Meta

Journal des traducteurs

Translators' Journal

\title{
L'interprétation de conférence en tant que profession et les précurseurs de l'Association Internationale des Interprètes de Conférence (AIIC) 1918-1953
}

\section{Walter Keiser}

Volume 49, numéro 3, septembre 2004

L'histoire de la traduction et la traduction de l'histoire

History of Translation and Translation of History

URI : https://id.erudit.org/iderudit/009380ar

DOI : https://doi.org/10.7202/009380ar

Aller au sommaire du numéro

Éditeur(s)

Les Presses de l'Université de Montréal

ISSN

0026-0452 (imprimé)

1492-1421 (numérique)

Découvrir la revue

Citer cet article

Keiser, W. (2004). L'interprétation de conférence en tant que profession et les précurseurs de l'Association Internationale des Interprètes de Conférence (AIIC) 1918-1953. Meta, 49(3), 576-608. https://doi.org/10.7202/009380ar

\section{Résumé de l'article}

Alors que l'interprétation remonte à l'après-Babel, l'interprétation de conférence en tant que profession n’a qu'un siècle. Les grands consécutivistes pionniers de la profession, l'avènement de l'interprétation simultanée et sa percée au procès de Nuremberg et aux Nations unies, l'extension de la pratique de l'interprétation au monde entier, la formation des interprètes, leurs conditions de travail, l'indispensable organisation de la profession, voilà l'objet de cet article enrichi de nombreuses anecdotes et de souvenirs de l'auteur, interprète lui-même depuis 1948. 


\title{
L'interprétation de conférence en tant que profession et les précurseurs de l'Association Internationale des Interprètes de Conférence (AIIC) 1918-1953
}

\author{
WALTER KEISER \\ Association Internationale des Interprètes de Conférence (AIIC) \\ w.keiser@webcreatif.ch
}

\begin{abstract}
RÉSUMÉ
Alors que l'interprétation remonte à l'après-Babel, l'interprétation de conférence en tant que profession n'a qu'un siècle. Les grands consécutivistes pionniers de la profession, l'avènement de l'interprétation simultanée et sa percée au procès de Nuremberg et aux Nations unies, l'extension de la pratique de l'interprétation au monde entier, la formation des interprètes, leurs conditions de travail, l'indispensable organisation de la profession, voilà l'objet de cet article enrichi de nombreuses anecdotes et de souvenirs de l'auteur, interprète lui-même depuis 1948.
\end{abstract}

\begin{abstract}
Conference interpreting as a profession was born at the end of the First World War. Practised first by a small number of extraordinary consecutive interpreters, its development and spread worldwide came with the breakthrough of the technique of simultaneous interpretation at the Nuremberg Process and in the United Nations. The training of the interpreters, their working conditions, the first attempts to organize the profession are described with a flourish of anecdotes and historic memories by the author whose career as an interpreter started in 1948.
\end{abstract}

\section{MOTS-CLÉS/KEYWORDS}

interprétation de conférence, interprétation simultanée, formation et conditions de travail, précurseurs de l'organisation professionnelle, anecdotes historiques

\section{Introduction}

On peut dire que si l'interprétation de conférence en tant que profession a vu le jour à la fin de la Première Guerre mondiale, l'idée d'organiser cette profession ne s'est précisée et réalisée qu'après la Seconde Guerre mondiale. Pour bien comprendre les principes directeurs et la structure de l'AIIC, il est utile de rappeler qui étaient les pionniers de l'interprétation de conférence au temps de la Société des Nations et de se livrer ensuite à une analyse assez poussée de la situation de la profession à l'issue de la guerre. Nous procéderons par matières selon la systématique suivante:

- Les pionniers de l'interprétation de conférence (1918-1945).

- L'après-guerre (1945-1953).

- Les interprètes: Nuremberg, Nations unies, marché privé.

- L'évolution des modes d'interprétation avec l'essor de la simultanée: précurseurs, Nuremberg, ONU, ailleurs. 
- Les conditions de travail dans les différents circuits : organisations internationales, marché privé, activités accessoires.

- La formation des interprètes: écoles, autres types de formation, litige.

- La dispersion géographique, circuits, marchés de l'interprétation.

- Les associations et groupements d'interprètes précurseurs de l'AIIC ou contemporains.

Il va de soi qu'une description complète de cette évolution et l'énumération de tous les interprètes qui ont marqué cette période dépasseraient de loin le cadre de cet ouvrage. Mais nous espérons, par les exemples choisis, réussir à brosser un tableau fidèle des situations et des pratiques qui menèrent à la création de l'AIIC, voire rendirent celle-ci indispensable si l'on voulait assurer l'avenir de la profession.

\section{Les pionniers de l'interprétation de conférence (1918-1945)}

Les hommes et les quelques rares femmes qui faisaient de l'interprétation de conférence leur métier, du moins partiellement, étaient très peu nombreux. Il y avait quelques fonctionnaires (à la Société des Nations), à peine une douzaine de free-lance, d'après André Kaminker, mais ceux-ci exerçaient presque tous des activités accessoires et ils étaient tous arrivés à la profession par d'autres métiers. Quelques-uns, après un passage rapide par l'interprétation, reprenaient des carrières diplomatiques ou administratives. Ils étaient tous d'excellents linguistes et presque tous dotés d'une formidable personnalité. En voici quelques-uns.

Le premier à marquer l'histoire de la profession d'interprète de conférence fut Paul Mantoux, professeur, homme de lettres, interprète. Une notice biographique nous apprend que, délégué du premier ministre de l'Armement de la France à Londres, au cours de la première guerre mondiale, il gagna la confiance du premier ministre de Grande-Bretagne, Lloyd George, ainsi que de Lord Asquith, qui réclamèrent sa présence lors de leurs rencontres avec leurs homologues français. Paul Mantoux devint en juin 1918 l'interprète du Conseil suprême interallié, puis du conseil des Quatre, en mars 1919. Les membres de ce conseil négocièrent le traité de Versailles. Ce fut Paul Mantoux qui définit admirablement l'essence de notre métier en écrivant en 1946 un article pour L'Interprète sous le titre «Interpréter, c'est d'abord comprendre».

Jean Herbert, qui considérait Paul Mantoux comme son mentor, eut la première expérience d'interprète de conférence en 1917, lorsque, rentré du front pour un congé, il fut engagé pour accompagner M. Thierry, ministre français des Finances, et le gouverneur de la Banque de France à Londres pour négocier un emprunt. La réunion se tint dans la maison de Lloyd George, "around the breakfast table, to the accompaniment of ham and egg. I am grateful», dit Jean Herbert, «that my interpretations were not recorded, because if I heard them now I would certainly blush. However, it was the best that could be done at the time and, strange as it may sound, it was appreciated.»

Aux négociations suivant l'armistice - il n'y eut pas de Conférence de la paix à proprement parler - il y avait une douzaine d'interprètes travaillant en anglais et en français. Les négociations avec les Allemands se firent à part. Les interprètes allemands étaient en général de jeunes officiers connaissant parfaitement le français ou l'anglais. C'est là que peu à peu, on passa de l'interprétation "phrase par phrase » à une véritable interprétation consécutive, les interprètes travaillant en équipe de deux, interprétant « verbatim speeches which occasionally lasted well one hour». Jean Herbert 
n'était pas seulement un des doyens de cette génération d'interprètes, il était l'auteur de plusieurs ouvrages sur les religions orientales et, bien sûr, du fameux Manuel de l'interprète (1952), livre de chevet de quiconque entendait enseigner l'interprétation. La profession lui doit beaucoup. Après la Deuxième Guerre mondiale, c'est à lui qu'on s'adressa pour diriger les premiers services d'interprétation dans le cadre des Nations unies. Son expérience et sa formidable personnalité lui permirent de s'imposer et de faire placer d'emblée les interprètes de conférence à un niveau statutaire et de rémunération enviable. Écoutons Roger Glémet: "Je crois que les interprètes d'aujourd'hui ne savent pas tout ce qu'ils doivent à Jean Herbert, qui a tenu "la dragée haute" aux institutions et obtenu satisfaction totale.» Rappelons que Jean Herbert fut président de l'AIIC de 1966 à 1969.

Certains interprètes free-lance de cette époque avaient des clients réguliers, mais pas exclusifs: la Banque des règlements internationaux eut recours à Michaelis, puis, pendant de nombreuses années, à Antoine Velleman. Le second, avec ses quatre langues d'aboutissement, interprétait pour un nombre très varié d'organisations tout en poursuivant une remarquable carrière de professeur de langues - il avait reçu la citoyenneté d'honneur suisse comme auteur de la première grammaire de langue romanche. William O'Davoren était partenaire d'un cabinet d'avocats à Genève et l'auteur du premier ouvrage sur l'organisation technique et la procédure parlementaire des conférences internationales (Post-War Reconstruction Conferences, 1943).

Les premières utilisations de l'interprétation simultanée eurent lieu pendant les années 1920, à Genève (Bureau international du travail) et en URSS (VIème Congrès de l'Internationale communiste). La nouvelle technique se heurta à l'opposition des grands «consécutivistes». Nous en parlerons dans la section consacrée aux modes d'interprétation.

Dans l'entre-deux guerres, les conditions de rémunération des fonctionnaires dépendaient du grade qu'ils occupaient, celles des free-lance étaient en général librement négociées, rarement le fruit d'une concertation. Quant aux conditions de travail (composition des équipes, charge de travail, etc.), elles n'étaient spécifiquement définies par rapport à l'interprétation de conférence, ni pour les fonctionnaires, ni pour les free-lance. On assistait fréquemment à ce qui, aujourd'hui, serait considéré comme des prouesses, pour ne pas dire des folies, par exemple des discours en consécutive debout d'une heure entière. L'auteur se souvient qu'à l'âge de 14 ans, à Caux où il accompagnait sa mère à une réunion de trois jours du Réarmement moral, après chaque discours un monsieur glabre et moustachu se levait, droit comme un «i », résumant le discours tour à tour pendant 5 minutes environ dans trois des quatre langues de la réunion (allemand, français, anglais, italien). La première fois, Madame Keiser expliqua: «Ça, c'est l'interprète. » Eh bien l'interprète en question était Antoine Velleman.

On pourrait parler longuement de ces grands anciens. Le phénoménal André Kaminker mériterait un article à lui tout seul, ainsi que les grands consécutivistes de la Société des Nations tels que Confino, Jacob, Lloyd, Margouliès, Mathieu, Royer et le colonel Wade. Nous en trouvons une excellente évocation dans l'ouvrage d'Henri van Hoof et dans la remarquable thèse de Jesús Baigorri-Jalón sur l'interprétation de conférence. Ce furent des personnalités admirées par les délégués et les journalistes de cette époque où, comme le dit si bien Baigorri-Jalón, «cette activité était davantage assimilée à un art qu'à une profession». Nous retrouverons plusieurs de ces interprètes après la Deuxième Guerre mondiale. 
À noter que Baigorri-Jalón consacre une partie de son ouvrage à une catégorie d'interprètes dont nous n'avons pas parlé jusqu'ici, mais qui, en vertu de la technique d'interprétation utilisée, peuvent être assimilés aux premiers interprètes de conférence. Ce sont «les interprètes des dictateurs, avec leur fidélité et identification au personnage principal, leur disponibilité en permanence et leur capacité de s'adapter à toutes sortes de situations d'interprétation». Un nom vient immédiatement à l'esprit, celui du Dr Paul Schmidt que certains qualifient un peu sommairement «d'interprète de Hitler». Erich Feldweg précise que Paul Schmidt était fonctionnaire aux Affaires étrangères dès avant l'avènement de Hitler et donc autant l'interprète de Stresemann. Comme de nombreux autres non-nazis il choisit de rester en service après 1933. Au demeurant, c'était un excellent interprète comme le lui attesta, par exemple, Sir Neville Henderson. Nous n'en dirons pas plus.

\section{L'Europe d'après-guerre}

Dans l'après-guerre immédiat, il s'agissait de reconstruire l'Europe en ruines, réorganiser les États, parfois retracer des frontières. Pour tout cela, il fallait négocier des accords, redéfinir des alliances, assurer la coopération. Il fallait se comprendre, donc surmonter les frontières linguistiques. En un mot : il fallait aussi des interprètes. Il n'y a là rien de nouveau. Comme la remarquable exposition sur l'histoire de la profession organisée dans le cadre de l'Assemblée de l'AIIC 1997 à Montréal nous l'a rappelé: «Il est clair que déjà en Grèce antique, dans la Rome antique, à chaque fois que les nations se confrontaient dans les guerres et à chaque fois que l'on se rencontrait pour parler de la paix, il y avait toujours, juste derrière le général ou derrière le diplomate, l'interprète.»

Plusieurs facteurs entraient en ligne de compte: Où trouver les interprètes? Comment faire face à la demande pressante? Quels moyens techniques mettre en œuvre?

\section{Les interprètes}

Antoine Velleman avait vu juste lorsqu'en 1941 il obtint, non sans mal, de l'Université de Genève la fondation d'une École d'interprètes en arguant que la demande serait considérable dans l'après-guerre immédiat. Les enseignants étaient en majorité les interprètes désœuvrés de la Société des Nations, les étudiants venaient en partie des camps de réfugiés en Suisse. Ainsi, les diplômés de l'École de Genève, s'ajoutant aux interprètes chevronnés de feu la Société des Nations - dont beaucoup, comme nous le verrons plus tard, furent repris dans les cadres d'interprètes des Nations unies - se trouvèrent engagés, qui au procès de Nuremberg, qui pour les grandes conférences intergouvernementales et non gouvernementales d'après-guerre, qui encore au service des gouvernements ou futurs gouvernements. Ils y rencontrèrent d'anciens réfugiés, déracinés mais forts en langues, culture et matières diverses et qui avaient appris à interpréter sur le tas, ou encore des interprètes sortis des services armés.

Le procès de Nuremberg, ou plutôt, les procès de Nuremberg - procès principal des grands criminels de guerre, procès de suivi: capitaines d'industrie, juristes, médecins et chefs de camps de concentration (1945-1947) - en fut un exemple. Le service d'interprétation fut placé sous la responsabilité de Léon Dostert, Lorrain 
d'origine, colonel de l'armée américaine et ancien interprète d'Eisenhower. Haakon Chevalier fut le second de Dostert. Le français Jean Meyer recruta les premiers interprètes de cabine française. Wolfe Frank fut le deuxième chef-interprète, Peter Ueberall, qui avait d'abord été «moniteur» - voir chapitre "Conditions de travail» ci-après - succéda à Frank. Tous les quatre étaient des anciens des services armés, comme d'ailleurs un nombre important de la trentaine d'interprètes qui servirent à Nuremberg. D'autres furent recrutés par leur gouvernement, tels Elisabeth Heyward, Marie Rose Waller, Génia Rosoff, Marie France Rosé (Skuncke), Helga Lund, Stefan Priacel. Un groupe provint de l'École d'interprètes de Genève: Stefan Horn, Frédéric (Fred) Treidell, Patricia Jordan (van der Elst), Armand Jacoubovitch, Peter Less, Norbert Berger. Nous ignorons le chemin qui amena d'autres à Nuremberg, par exemple Eric Simha, Ursula Conventry, Georges Klebnikof, qui sortait de HEC à Paris, Edouard Roditi, George Wassilchikof, Nora Saxe, Jimka Pachkof. André Kaminker y fit un passage rapide. L'auteur, alors étudiant de première année, se souvient du test organisé fin 1945 avec un équipement de simultanée improvisé à l'Aula de l'Université de Genève par le colonel Léon Dostert en vue du recrutement du deuxième groupe d'interprètes pour Nuremberg. Les interprètes russes enfin, en uniforme et rattachés aux services armés, vivaient dans des quartiers réservés aux soviétiques et restaient à part.

Des témoignages reçus, nous savons que l'ambiance au procès même était tendue, le Palais de justice lugubre, la responsabilité énorme. Seuls deux interprètes, Haakon Chevallier et Edouard Roditi, apportaient une expérience professionnelle en interprétation simultanée. Selon le cas, les arrivants pouvaient s'y mettre graduellement, en passant d'abord par le service de traduction, d'autres furent mis en audience du procès presque immédiatement. La responsabilité et le fond dramatique des audiences s'avérèrent insupportables pour quelques interprètes, eux-mêmes victimes du nazisme ou enfants de victimes. Ainsi, Armand Jacoubovitch et Margrete Abrahams passèrent à la traduction.

Malgré cette tension et malgré le fait qu'il y avait un roulement incessant de partants et d'arrivants dans les équipes, l'esprit de camaraderie était en général excellent. Comme le dit Marie-France Skuncke, le soir il fallait se détendre, "on s'est beaucoup amusé». Les interprètes étaient logés soit à l'hôtel, soit dans des villas réquisitionnées. Leur rémunération dépendait de leur rattachement: ceux qui avaient la chance de dépendre des Américains et d'être payés en dollars s'en sortaient très bien, ceux relevant d'autres gouvernements ou forces armées nettement moins.

Les interprètes de Nuremberg et l'interprétation simultanée ont-ils été officiellement appréciés? Dans son interview avec Rosalinda (Rosie) Meza-Steel, Peter Ueberall nous dit son regret que dans aucun document officiel du procès on ne parle de l'interprétation ou des interprètes, ni en bien, ni en mal. Pour les responsables du procès, pense-t-il, «cela faisait tout simplement partie du système» et tant que celuici marchait tout était bien. Pourtant, nous avons trouvé une autre interview où Sir Hartley Shawcross, attorney general au procès, déclare que «the interpreters were using the simultaneous method, that is to say they write down nothing but interpret right away by telephone. I am of the opinion that simultaneous interpretation should be adopted at all international meetings as it saves so much time and interpreting being, of course, absolutely essential at any meeting of an international character ».

Après le procès, certains interprètes de Nuremberg quittèrent le métier, revenant 
à leurs occupations civiles ou militaires antérieures. D'autres restèrent interprètes et essaimèrent: Stephan Horn suivit Dostert aux États-Unis et prit la direction du Department of Translation and Interpretation de la School of Languages and Linguistics, fondée par le même Léon Dostert à l'Université de Georgetown (Washington, DC). Jean Meyer, retourné au Quai d'Orsay, devint chef-interprète du Comité intérimaire de la Communauté européenne de défense (1952-1954). Fred Treidell fut nommé «Head of press intelligence and Special Projects » du European Recovery Program (Plan Marshall) et devint plus tard chef-interprète de l'Agence européenne de productivité. D’autres encore furent amenés par le colonel Dostert aux Nations Unies à New York pour y faire accepter l'interprétation simultanée, tels Georges Klebnikof et Eric Simha.

Ce qui nous amène aux Nations unies. Sans vouloir écrire ici l'histoire des institutions de l'ONU, il nous paraît intéressant de rappeler qui étaient les interprètes entre la fin de la Société des Nations et la première Assemblée de New York. Nous nous appuyons pour cela sur des témoignages d'interprètes, notamment de Roger Glémet et surtout de Georges Thorgevsky, un des rares survivants de ce groupe.

La conférence de San Francisco de 1945 et la Commission préparatoire avaient arrêté les principes de base et les structures essentielles de l'ONU et l'on s'apprêtait à tenir la première Assemblée générale, fixée pour janvier et février 1946 à Londres.

La plupart des interprètes et des traducteurs de la Section linguistique de cette Assemblée, responsables compris, étaient des anciens de la Société des Nations, notamment Georges Mathieu, interprète de grande valeur, et son adjoint, le Britannique bilingue originaire des Îles anglo-normandes Le Bosquet. M. Demolon, qui fut professeur agrégé du lycée Condorcet, dirigea les activités des traducteurs. Jean Herbert, qui avait déjà dirigé l'équipe d'interprètes à San Francisco, était à nouveau en charge. Les interprètes étaient en majorité d'origine française, exceptés Daniel Hogg, Britannique marié à une Allemande, qui parlait un anglais superbe et connaissait le russe, et le commandant Lambert-Lammond, qui s'orienta plus tard vers l'Assistance technique et l'Aide au développement. Il y avait en outre: Georges Kaminker et R. Confino, précis et précieux, N. Teslenko, Russe, qui venait de terminer ses études de droit après avoir passé 4 ans en Allemagne comme prisonnier de guerre, Georges Thorgevsky, autre russisant «découvert» par Jean Herbert, R. Cru, journaliste mais qui travaillait aussi comme interprète, le philologue G. Margouliès, un des plus âgés, seul interprète maîtrisant les cinq langues des Nations unies et, les plus spectaculaires, André Kaminker et Jean Herbert lui-même. Une équipe de dix interprètes pour l'Assemblée des Nations unies, douze pendant les moments de grande presse lorsque Mathieu et Le Bosquet venaient prêter main forte aux autres. Tout le travail se faisait en interprétation consécutive.

L'avenir new-yorkais du service d'interprétation fut assuré dès l'Assemblée de Londres. Georges Thorgevsky raconte que «le Secrétaire Général Trygve Lie, élu le $1^{\text {er }}$ janvier 1946, pria Jean Herbert de constituer un service d'interprètes permanents et l'équipe de la première Assemblée fut conservée presque au complet». Elle fut renforcée à New York, notamment, par l'arrivée des simultanéistes du colonel Dostert.

Les interprètes des premières années d'après-guerre connurent des sorts divers. Certains accédèrent à des postes de responsabilité, en qualité de chef-interprètes, d'autres, bien que brillants interprètes, quittèrent la profession à plus ou moins longue échéance, pour se consacrer à d'autres activités. Quelques exemples: Constantin 
Andronikof, détaché par le Quai d'Orsay, devint chef-interprète de l'OECE, Hans Jacob de l'UNESCO, Kouindji du BIT, Cecil Biass de la FAO. Deux des interprètes de l'Assemblée de l'ONU de Londres devinrent chef-interprètes en Europe, A. Kaminker au Conseil de l'Europe et R. Confino à l'Office des Nations unies de Genève. Boris Melikoff succéda à Andronikof comme chef-interprète de l'OECE, suivi par Paul Unwin, tous deux appelés à jouer un rôle important pendant la mise en place de l'AIIC. Deux anciens interprètes militaires accédèrent tout naturellement au poste de chef-interprète de l'OTAN, Max Lemansois-Field et du SHAPE, Guy Gribble. Boris Desfontaines fut nommé chef-interprète au Conseil de coopération douanière. Le sort de Haakon Chevallier mérite d'être signalé: rentré aux États-Unis, il fut pris dans la tourmente du maccarthysme et écrivit le remarquable ouvrage sur «l'affaire Oppenheimer». Destin glorieux et tragique, celui de Cyrille (Kyra) Borowski, originaire de Leningrad, interprète remarquable qui se distingua à l'UNESCO, mais fut surtout connu sous son nom de plume, Edmond Cary, auteur d'ouvrages sur la traduction qui firent date, co-fondateur et secrétaire général pendant dix ans de la Fédération internationale des traducteurs. Borowski mourut tragiquement lors de la catastrophe aérienne d'un vol d'Air India au Mont-Blanc en 1966. Jean-François Rozan, jeune prodige de la filière des Nations unies et pédagogue exceptionnel, compléta le Manuel de l'interprète de Jean Herbert par son ouvrage sur la Prise de notes en interprétation consécutive (1956) pour quitter peu après la profession en se lançant dans l'hôtellerie et l'immobilier.

Les interprètes de cette époque étaient souvent des personnalités hautes en couleur, certains auteurs parlaient même, pour les décrire, de «sociological freaks». Et chaque nouvelle conférence était un lieu où l'on faisait connaissance, pour se retrouver par la suite, à composition variable, dans tous les coins de l'Europe. Un exemple suffira: le Congrès mondial du bois de Helsinki en 1949, premier congrès international de l'après-guerre en Finlande, organisé sous le patronage de la toute nouvelle FAO, (chef-interprète: Marie Ginsberg). Le tout en consécutive debout, anglaisfrancais et vice-versa, quelques séances avec le russe en plus. Il y avait là, entre autres, Teddy Pilley avec son scooter, le boulimique Adam Richter qui par la suite, fit œuvre de pionnier pour l'interprétation de conférence en Israël, Guy Piquemal, interprète virtuose qui passa ensuite à l'UNESCO, Hélène Pfaendler, toujours au pas de course, le «citoyen» Imbert, encyclopédie technique vivante: il ne connaissait pas seulement tous les termes en trois langues, il savait en plus comment cela marchait. Pourquoi «citoyen»? Parce que, enthousiasmé par la victoire des Soviétiques, tout Français qu'il était, il demanda à l'Ambassade soviétique immédiatement après la guerre à obtenir un passeport soviétique et il l'obtint en un temps record. Il y avait aussi l'aristocratique $\mathrm{H}$. Colin du Terail. À la fin du congrès, l'auteur vécut une aventure avec Teddy Pilley, la première d'une longue série: de retour de deux belles journées à $280 \mathrm{~km}$ au nord-est de Helsinki chez le propriétaire d'une grande scierie, père d'une des ravissantes secrétaires du congrès qui nous y avait invités, nous roulions à quatre en Citroën 11 CV de location, Teddy au volant. Il devait prendre l'avion pour Londres le soir même. La conversation allait bon train. On roulait et roulait, jusqu'au moment où nous nous trouvâmes entourés d'une patrouille de gardes-frontière soviétiques. Teddy, tout à la conversation, avait pris trop sur la gauche (sud-est). Un des interprètes, descendant de parents russes blancs n'osa pas ouvrir la bouche. Les explications de Teddy en polonais furent infructueuses. Finalement, notre collègue russe 
parla. Il nous fallut 36 heures, via Moscou-Leningrad-Ambassade soviétique à Helsinki pour être libérés. Ce qui nous sauva, c'est que l'ambassade soviétique confirma que nous étions bien des interprètes et que nos noms figuraient sur la liste des invités de la réception que la délégation soviétique avait offerte au Congrès mondial du bois l'avant-dernier soir. Épilogue: Teddy rata son avion.

Il serait très intéressant de retracer en détail la personnalité et la carrière d'autres collègues de cette époque. L'espace nous manque, pourtant le temps presse: nombreux sont les ancêtres de la profession qui nous ont quittés. Avec certains d'entre eux nous avons perdu des trésors d'anecdotes et de témoignages.

Deux points capitaux à retenir encore sous ce titre, valables pour tous les interprètes occidentaux :

- Dès les débuts de la profession, il n'y eut pas de différence de statut ou de rémunération entre interprètes hommes et femmes. Il est vrai que d'abord on y trouvait plus d'hommes que de femmes. Cela tenait probablement au fait que l'interprétation consécutive régnait souverainement et que les salles de réunion étaient rarement sonorisées. Il fallait avoir une voix qui porte et il ne fallait pas avoir peur de se lever pour parler devant des centaines de personnes. Mais très vite, les collègues femmes s'imposèrent et nous écoutions avec admiration les Jocelyne Brunet, Marie-France Skuncke et Elisabeth Hedinger (Meister) à côté des maîtres de la consécutive déjà mentionnés. Avec l'avènement de l'interprétation simultanée et des écoles d'interprétation, l'équilibre des sexes se rétablit rapidement.

- Partout on faisait confiance aux interprètes quelle que fût leur race, religion ou provenance géographique. On savait qu'on pouvait compter sur le secret professionnel. Une anecdote: fin 1948, le Dr Reichhold, directeur du service linguistique du ministère des Affaires étrangères du gouvernement Adenauer, vint à Genève pour recruter des interprètes. Il nous raconta qu'une question s'était posée: Où trouver des interprètes qualifiés? Le Dr Reichhold, qui connaissait la situation, avait dit à M. Adenauer : "Il n’y a plus de vrais interprètes de conférence en nombre suffisant en Allemagne. Ou bien ils sont morts, ou bien ils sont "persona non grata", s'étant trop mouillés avec le régime nazi. Il faut donc recruter ailleurs. » Là-dessus, il y eut des protestations vigoureuses au sein du gouvernement. On disait: «Mais quoi, des étrangers! Est-ce qu'on va pouvoir leur faire confiance?» C'est Adenauer lui-même qui répondit: «Messieurs, l'Allemagne a tout perdu, l'Allemagne n'a que la force de ses arguments. Nous devons veiller à ce que nos arguments portent à cent pour cent chez les négociateurs de l'autre côté de la table. Il nous faut donc trouver les meilleurs interprètes capables de nous en assurer, d'où qu'ils viennent. D'ailleurs ", - ajouta-t-il, «tous ces interprètes sont tenus au secret professionnel». Un mot historique pour notre profession s'il en fut.

À propos de secret professionnel, on dit qu'André Kaminker, alors président de l'AIIC, était sensé prêter son concours à la rédaction du Code d'honneur. Il gribouilla sur un bout de papier et dit: «Le voilà, votre code d'honneur.» Sur la feuille il y avait une seule phrase: «L'interprète de conférence est tenu au secret professionnel.»

\section{Les modes d'interprétation}

Dans l'après-guerre immédiat, l'interprétation consécutive était à son apogée et de très longs discours interprétés en une fois n'étaient pas exceptionnels. Écoutons Elisabeth Meister: "Des interprétations consécutives de rapports de secrétaires généraux de plus d'une heure, à l'Union interparlementaire ou à l'ancienne Organisation internationale des organismes de tourisme... je m'en souviens» Ces longues interprétations consécutives produisirent parfois des effets cocasses. Elisabeth raconte: 
«En juin 1951 se tient à Lausanne la Conférence des P.E.N. Clubs, je travaille seule en consécutive pour l'anglais et le français. Le clou de la réunion est la conférence d'une philologue anglaise qui parle du mouvement des légendes d'est en ouest, de la diffusion des noms des personnes et des choses pendant les migrations des premiers siècles. Son exposé dure une heure, je prends des notes assidues, et lorsque je commence "ma" consécutive, elle quitte la salle, suivie bientôt des “anglophones". J'ai l'auditoire "francophone" pour moi toute seule, à peu près la moitié des participants. Je refais la conférence en français à partir de mes notes, en trois quarts d'heure...» Les grands consécutivistes brillèrent de tous leurs feux. Norman Langford, qui allait rejoindre les rangs des interprètes du Bureau international du travail en 1967, se souvient de son arrivée à Genève en 1948: "Quant à Herbert et Confino, je leur portais une admiration sans bornes alors que j'exerçais le métier de traducteur/procès-verbaliste aux Nations unies cette même année. Je me souviens d'avoir ainsi travaillé à des réunions où les interventions étaient accueillies dans le silence, tandis que les traductions avaient droit aux applaudissements des délégués.»

Les deux modes d'interprétation les plus anciens du monde, l'interprétation consécutive «phrase par phrase» et la «chuchotée», continuèrent à se pratiquer pendant toute cette période, souvent dans des conditions épouvantables, il est vrai : salles enfumées, bruits de fond massifs, "chuchotage » à 10 délégués ou plus. Quant aux nouveaux moyens techniques, ils furent imposés par l'explosion du nombre de réunions plurilingues nécessitant l'interprétation, les nations d'après-guerre n'admettant plus l'hégémonie du français comme langue diplomatique universelle. L'histoire de la simultanée mérite qu'on s'y arrête.

La technique de la simultanée fut utilisée sporadiquement entre les deux guerres. La question a été posée si les premières simultanées, ou concomitantes - nous avons aussi trouvé «instant translation » et "interprétation téléphonique - étaient de vraies interprétations simultanées ou une lecture simultanée de notes prises préalablement par plusieurs interprètes de consécutive. De la documentation entre nos mains remise par Kamal Annabi -, portant sur une interview entre M. Gordon Finlay et Gérard John en 1946 et d'un article de R. Durel, ancien chef de l'administration du BIT, il ressort que l'interprétation au moyen des appareils HUSHAPHONE FileneFinlay (brevet repris par IBM) était bel et bien une vraie simultanée. La première eut lieu dans la salle du Conseil général à l'occasion de la conférence annuelle 1927 du Bureau international du Travail, après des essais au BIT même en 1926, autorisés par le directeur Albert Thomas en personne. Les interprètes travaillaient en pleine vue des délégués et non pas, comme cela a été dit, cachés derrière des buissons. De même, l'interprétation pratiquée par André Kaminker avec un équipement rudimentaire à l'occasion du discours de "prise de pouvoir» de Hitler à Nuremberg en 1933 était également une vraie simultanée. Autre rappel historique. La première utilisation de l'interprétation simultanée en Union soviétique eut lieu à l'occasion du VIème Congrès de l'Internationale communiste en 1928. Une image du journal Krasnaya Niva (Champ rouge) montre les interprètes assis au fond de la tribune, un pesant «harnais » doté d'un microphone autour du cou. Ils n'avaient pas d'écouteurs et captaient le son directement de la salle. Par la suite, l'interprétation simultanée fut utilisée par-ci par-là, notamment lors de quelques congrès scientifiques ou techniques. Dans L'Interprète (1957, n 4) Magda van Emde Boas décrit le procédé utilisé en été 1935 à l'occasion du XVème Congrès international de physiologie à Leningrad (le «Congrès Pavlov»). 
Comment cette nouvelle technique a-t-elle été reçue par les délégués et par les interprètes de l'époque? Karla Dejean Le Féal nous a procuré des témoignages qui nous ont permis à la fois de confirmer les dates de la première utilisation de l'interprétation simultanée à l'OIT et d'enregistrer la réaction des utilisateurs. En voici deux:

- Lettre du marquis G. Paolucci de Caltoli Barone, sous-secrétaire de la Société des Nations, à M. Ake W. Hj. Hammarskjoeld, greffier de la Cour permanente de justice de La Haye (9 novembre 1927). «[...] this system of interpreting is, of course, in many ways much more difficult than the ordinary method as the interpreter has to listen to one sentence while translating the precedent one. It was thought at first that this difficulty might prove insurmountable, but several of the interpreters at the Labour Conference achieved excellent results.»

- Les passages suivants sont de la plume d'un des grands consécutivistes de la Société des Nations, Georges Mathieu, qui, s'adressant à M. Demolon, chef du Service des traducteurs et interprètes français de la SdN (3 septembre 1930), fait à la simultanée un procès en règle. Ayant assisté à une réunion desservie par les interprètes du Bureau international du travail, Mathieu relate ses impressions. «[...] entendant assez mal l'allemand, je voulus utiliser l'interprétation téléphonique pour prendre des notes sur les discours allemands, mais je dus bientôt y renoncer complètement. Ceci ne comporte, bien entendu, aucune critique pour mes collègues du BIT qui, soit en français, soit en anglais, traduisaient les discours aussi bien qu'il est possible avec un système aussi défectueux. L'imperfection est inhérente au fait, pour les interprètes, de devoir parler en même temps que l'orateur. Cela les oblige à sauter des phrases du discours [...] ils n'ont pas la possibilité de résumer intelligemment ni de choisir les passages sautés. Il se peut que la phrase prononcée par l'orateur pendant qu'ils traduisent et qu'ils n'entendent pas, par conséquent, soit justement une des plus importantes. L'effet est incohérent et il est absolument impossible de suivre une idée ou un développement dans son entier.» Passant aux délégués, Mathieu continue: «[...] je suis certain qu'un délégué qui ne connaît absolument pas l'autre langue officielle n'entendra que des mots mais il ne pourra pas suivre l'ensemble d'un exposé [...] pratiquement, il sera exclu de toute une partie des travaux de l'Assemblée». Plus tard Mathieu craint «qu'on pourrait difficilement songer à interrompre $\mathrm{M}$. Briand, par exemple, au milieu d'un discours pour lui demander de parler plus lentement à cause de l'interprétation téléphonique». Ensuite il voit juste en disant: «[...] on peut craindre que l'adoption du système téléphonique n'ouvre la porte à la demande de reconnaissance d'une ou de plusieurs langues officielles supplémentaires, ce qui entraînerait des dépenses infiniment plus grandes encore que celles des installations de matériel... Quant au point de vue personnel des interprètes (de la SdN, W.K.), je n'y reviendrai pas sauf pour signaler l'insalubrité évidente des petites boîtes à l'intérieur desquelles ceux-ci doivent parler.» En conclusion de sa diatribe, Georges Mathieu déclare sans ambages: «[...] je ne désire pas me prêter à cette expérience, ce n'est naturellement pas par mauvaise volonté ou désir de faire obstacle au progrès, mais bien parce que j'ai acquis la conviction qu'il n'y a pas là un véritable progrès. Je ne tiens pas à faire ce que je considère sincèrement comme du mauvais travail. Cela irait à l'encontre de ma conscience professionnelle et je ne voudrais pas me prêter à une innovation que j'estime contraire à l'intérêt des délégués et à la bonne marche des travaux de l'Assemblée.»

Cette attitude fut partagée par la majorité des interprètes de cette époque et il fallut attendre la fin de la Deuxième Guerre mondiale pour assister à la percée de la simultanée. En effet, ce fut au procès de Nuremberg, avec ses quatre langues et ses réunions fortement médiatisées, que l'interprétation simultanée s'imposa d'emblée. 
Le colonel Dostert, prouvant par un simple calcul qu'avec trois consécutives après chaque intervention le procès durerait des années, eut vite gain de cause. L'équipement installé dans la grande salle des audiences par le major Vincent du Signal Corps fut celui d'IBM, système Filene-Finlay. Il incluait un dispositif d'alerte en cas de difficultés rencontrées par les interprètes, signalées par le «monitor» et actionné par le président de la Cour. En cas de difficulté de routine - «orateur parle trop vite», «veuillez faire répéter, svp» - une lampe jaune s'allumait; en cas de crise aiguë, une panne totale par exemple, une lampe rouge, événement rarissime d'après Peter Ueberall. Le moniteur veillait entre autres à la présence des interprètes en début de séance et s'occupait de tout ce qui pouvait leur être utile pendant leur travail. Les «cabines» étaient installées directement sur le côté des bancs des accusés, formant quatre compartiments séparés par des vitres - « un vrai aquarium», nous dit MarieFrance Skuncke, - l'isolation entre les compartiments était minime. Comme il n'y avait qu'un microphone (une grosse boule ronde) pour les trois interprètes occupant la cabine - un interprète par la langue de départ -, cela aurait pu être gênant, mais, comme nous raconte Fred Treidell, «la concentration requise était telle que les interprètes se collaient le micro directement devant la bouche et ne se sentaient guère gênés par le manque d'insonorisation ». Au point de vue technique, le système, bien que lourd par rapport à ce qu'on connaît aujourd'hui, donna parfaitement satisfaction malgré une définition du son insuffisante. Voilà ce qui ressort entre autres des témoignages enregistrés lors d'une cérémonie organisée par la Commission des interprètes permanents de l'AIIC à Bruxelles en août 1992 pour rendre hommage aux pionniers de l'interprétation simultanée au procès de Nuremberg.

Venons-en aux Nations unies et aux institutions spécialisées. Dès avant Nuremberg, l'interprétation simultanée fut pratiquée de l'autre côté de l'Atlantique, à la Conférence des États alliés et neutres de l'Organisation internationale du travail à Philadelphie en avril 1944. Edouard Roditi, charmant conteur et doté d'une culture générale phénoménale, nous en parle: «The conference was held at Temple University. Simultaneous interpretation was used only for the General Assembly, which took place in a hall that provided no booths for the interpreters, who were placed in a dark and airless basement beneath the platform where the presidium sat and to which the speakers also came. The interpreters were therefore unable to see any of the speakers and could barely hear them on account of the constant shuffling of feet above their heads. Nor could they be heard at all clearly, since they were not separated from each-other by any partitions. » Il n'était pas surprenant dans ces circonstances que les avantages de l'interprétation simultanée ne fussent guère évidents pour les participants. C'est peut-être une des raisons pour lesquelles la simultanée ne fut pas utilisée à la conférence de San Francisco des Nations unies.

Pour l'introduction de la simultanée aux Nations unies à New York, nous devons à nouveau l'essentiel de l'information à Georges Thorgevsky. "À la première Assemblée des Nations unies à Londres les interprètes ne travaillaient qu'en consécutive. Mais pour une organisation dotée de cinq langues officielles, la consécutive posa des problèmes. Dès lors que l'ONU n'utilisait pas encore l'enregistrement sonore, il était nécessaire de donner une interprétation complète, deux même, en anglais et en français, lorsque l'orateur avait parlé en russe ou en espagnol.»Vers la fin de cette assemblée, M. Manouilsky, délégué de l'Ukraine et président du comité chargé d'étudier les méthodes et les plans de travail fit une proposition que Thorgevsky cite comme suit: 
«Pendant des siècles, le français a été la langue diplomatique internationale et depuis la SdN nous avons aussi l'anglais. Mais ce n'est pas assez. Bien des pays sont empêchés de se faire entendre par leurs meilleurs représentants parce que ceux-ci ne connaissent pas ces langues. Il faut corriger cela et justement, en ce moment-même, à Nuremberg, se déroule la simultanée dans plusieurs langues. Il faut absolument envoyer là-bas un groupe d'observateurs, délégués, techniciens, interprètes, pour voir comment ça marche et adopter éventuellement cette méthode.» Cette proposition fut approuvée à mains levées. En août 1946, nous dit Thorgevsky, « une petite équipe composée d'interprètes "simultanés" ayant fait leurs preuves à Nuremberg, conduite par le colonel Dostert, vint travailler à Lake Success, où les Nations unies avaient installé leur siège provisoire. Essayée par l'Assemblée à l'automne 1946, la simultanée se révéla pleine de promesses et il y eut dès lors deux sections d'interprétation distinctes, rivales même.» Les grands consécutivistes craignaient une perte de qualité difficulté d'assurer le contrôle de qualité si l'orateur et l'interprète parlent en même temps -, mais aussi une perte de prestige à devoir se cacher dans des cabines au fond de la salle - «nos amis derrière le rideau de cristal», comme disait l'ambassadeur Vyshinsky. Mais l'année d'après, à l'Assemblée de 1947 à Flushing Meadows, ce fut le succès: les «jeunes turcs », recrutés par Dostert étaient à la hauteur, en anglais, français, russe, espagnol et chinois. Les Georges Klebnikof, Jean-François Rozan, Eric Simha, Sylvain Lourié et surtout, pour le grand public, l'incollable George Sherry avec ses interprétations haute vitesse - il a été chronométré à 240 mots la minute des discours enflammés de Vyshinsky eurent autant de succès qu'à l'époque de la Société des Nations les grands discours à effets de manche de Kaminker ou les merveilles de précision de Jean Herbert, sans parler du phénomène Roger Glémet qui, resté excellent consécutiviste, devint virtuose en simultanée au point que nos collègues procès-verbalistes disaient de lui que c'était le seul interprète dont les discours pouvaient être imprimés sans révision.

En 1947-1948 eurent lieu quatre autres grandes conférences de la famille des Nations unies, avec interprétation simultanée, où les simultanéistes de l'ONU-New York se retrouvèrent avec des free-lance européens, dont Jean Back et Alexandre Bernstein (futur président de l'AIIC, 1978-1981):

1. La Conférence de Londres sur les tarifs et le commerce (ICITO, plus tard GATT) à Church House.

2. La Conférence générale de l'UNESCO de Mexico (1947) avec une équipe d'interprètes pour la consécutive et une autre pour la simultanée.

3. La Conférence-fleuve (6 mois si nos renseignements sont exacts) de l'Union internationale des télécommunications à Atlantic City où plusieurs jeunes diplômés de l'École de Genève firent leurs premières armes. Claire Tolnay, venue des USA comme traductriceréviseur, y fut promue interprète-chuchoteuse, puis formée à la va-vite par le State Department et expédiée à la $1^{\text {ère }}$ Conférence panaméricaine à Bogota, gravement perturbée par un "pronunciamiento ». Le jeune Eric Simha, qui avait eu une activité éphémère comme speaker et «bruiteur» à la radio américaine, se couvrit de gloire en imitant au micro une attaque aérienne. Cela lui valut d'être renvoyé sine die.

4. La Conférence du Comité provisoire des fréquences de l'UIT à Genève, prévue pour 6 mois et qui allait durer deux ans, dont Valérie Bouladon nous raconte que «les séances de nuit commençaient à 21 heures et duraient souvent jusqu'à 5 heures du matin, on ne s'en plaignait pas, il n'était pas question de se faire remplacer ni de mettre trois interprètes par cabine». 
Retour à New York. Après Flushing Meadows, raconte Thorgevsky, «la fusion des deux sections fut décidée et il n'appartint ni à Jean Herbert ni au colonel Dostert de prendre la direction du service unifié : ce fut Georges Rabinovitch qui fut choisi. Jean Herbert fut affecté à Genève (ce qui le ravit) et Dostert se consacra à l'Université de Georgetown. M. Adrain Pelt, secrétaire général adjoint demanda à tous les interprètes de devenir "amphibies", c'est-à-dire d'étudier celle des deux méthodes qu'ils n'avaient pas encore pratiquée.»

Pourtant, l'interprétation consécutive résista vaillamment. Aux Nations unies, le Conseil de sécurité la conserva, les déclarations des membres du Conseil étant toutes traduites en consécutive alors que celles des représentants de pays non membres du Conseil, ou celles des témoins, n'étaient traduites qu'en simultanée. Dans d'autres organisations aussi la simultanée fut introduite aux assemblées alors que la consécutive fut maintenue encore longtemps aux autres réunions, pour des raisons de contrôle ou pour des raisons budgétaires. Il en était ainsi pour l'OECE.

De plus, on vit des systèmes hybrides combinant les deux modes d'interprétation dans une même réunion. Deux exemples:

- La «simultanéo-consécutive» pratiquée à la conférence de la Fédération syndicale mondiale (Londres, sept. 1945) et reprise, après la grande scission du monde syndicaliste, à la réunion préparatoire (Genève, été 1949) de la Confédération internationale des syndicats libres, fondée à Church House, Londres, fin 1949. À Genève, il y eut cinq langues: anglais, français, allemand, italien et "scandinave», et cinq interprètes. Les participants s'étaient répartis en cinq groupes linguistiques dans la grande salle des fêtes. Après chaque discours, quatre interprètes travaillaient simultanément en consécutive, chacun parlant à son groupe. Lorsqu'ils avaient fini, ils levaient le bras et le président donnait la parole à l'orateur suivant. La seule situation où il fallait une double consécutive était après un discours en langue scandinave, l'interprète «scandinave» étant seul à pouvoir assurer le retour vers l'anglais.

- La «consécutivo-simultanée». Au Conseil de l’Europe, passé à la simultanée pour ses deux langues officielles d'alors, l'anglais et le français, la délégation allemande, pendant quelques années, amenait ses propres interprètes aux sessions de l'Assemblée et l'on vit ainsi les Rappeport (Longet), Longerich et Keiser produire, du centre de l'hémicycle, des discours en consécutive, d'allemand en français ou en anglais, repris en simultanée par leurs collègues du Conseil de l'Europe.

Un aspect technique mérite d'être signalé: dès le début, les équipements d'interprétation simultanée, aussi artisanaux fussent-ils, permettaient l'interprétation en relais. En effet, du moment où il n'y avait souvent qu'un interprète par langue, le relais s'imposait. Il est vrai que lorsque l'installation n'avait ni écouteurs, ni cabines, le relais posait un problème. Il fallait vraiment dresser l'oreille pour saisir ce que disait le «collègue-pivot» assis trois places plus loin à la même table, tous les autres interprètes parlant en même temps que lui! En outre, l'interprétation vers deux langues à partir d'un même micro - «le retour» - était pratique courante. Mais le «commutateur A/B» n'existait pas. Aussi fallait-il que, par exemple, les délégués écoutant le russe se branchent sur «original» lorsque l'interprète de la cabine russe se mettait à traduire un discours original russe en anglais ou, si la position «original» n'existait pas sur leur récepteur, ce qui était souvent le cas, ils devaient ôter leur casque et écouter en direct dans la salle.

L'essor de la simultanée était inéluctable. Des organisations non gouvernementales s'y mirent aussi: l'Union interparlementaire, la Chambre de commerce internationale, 
un nombre toujours croissant d'organisations syndicales. Et n'oublions pas les missions de productivités, organisées en Europe par l'Agence européenne de productivité, annexe de l'OECE, aux États-Unis par le State Department en collaboration avec les gouvernements européens. Danica Seleskovitch rappelle la formation assurée par le State Department pour les interprètes de tous les pays qui bénéficiaient du plan Marshall et arrivaient par dizaines au Centre de formation du State Department. Les Français, payés en France par l'AFAP (Association française de productivité), étaient formés à Washington par Edmund Glenn. Un bon nombre d'entre eux, dont Namy, Curtis, Pinhas, les Seleskovitch, les Umansky, etc. ont fait là leurs premiers pas. Danica Seleskovitch devint même formatrice d'interprètes à Washington et ses nombreux contacts avec des collègues des Amériques lui permirent par la suite d'œuvrer puissamment, en sa qualité de secrétaire exécutif, pour l'extension de l'AIIC aux Amériques. Au Parlement suisse, l'interprétation simultanée fut officiellement introduite début 1948, après une période d'essai, avec Ginette Bazelli (Schaefer), Elisabeth Hedinger (Meister), Anne-Marie Haenni (Zumstein), et Berthe Rundte (van Mansvelt), qui avaient suivi un cours spécial organisé à leur intention par Jean Royer au Palais des Nations sur équipement «Hushaphone». Au Parlement belge on pratiqua l'interprétation simultanée dès avant la guerre. Ainsi, en quelques années, la simultanée se répandit dans le monde entier et devint assez rapidement le mode d'interprétation prédominant.

Certains interprètes se lancèrent dans ce nouveau marché en offrant, soit des services de location d'équipement d'interprétation simultanée mobile, soit des services plus complets d'organisation de congrès. La première, à notre connaissance, fut Marie Ginsberg, interprète entre les deux guerres, puis bibliothécaire de la Société des Nations, puis directrice de Simulta, fondée début 1951. Marie Ginsberg, femme de cour et femme d'affaires, préféra rester en dehors des organisations professionnelles, mais elle eut le grand mérite d'offrir à un très grand nombre d'interprètes débutants leurs premiers engagements et l'astuce de les encadrer par des interprètes chevronnés tels que Jean Herbert, Pierre Lambert et d'autres. Épaulée efficacement par son technicien Dorier, elle garda son entreprise jusqu'à sa mort fin 1987, fidèlement aidée par Béatrice Neuenschwander et, les dernières années, par Viviane Vaucher. Mentionnons aussi Charly Vonwiller (SIORC) volant de congrès en congrès aux commandes de son Cessna. À Paris, à la même époque, il y eut la SIIS (Société internationale d'interprétation simultanée, une des premières, en 1954, à utiliser des transistors dans les récepteurs-radio), dirigée par Christopher Thiéry, Joseph Umansky et le technicien Phil Webster qui, comme beaucoup de ses collègues, fut pour les interprètes plus qu'un collaborateur, un ami connaissant si bien leurs problèmes. Et n'oublions pas les pionniers du «bidule» (équipement portatif ultra-léger sans cabines, ni écouteurs) qui, sans se constituer en entreprises apportaient leur matériel dans le monde entier. Ainsi les deux Britanniques Frank Barker «l'Africain », qui travaillait de préférence seul en simultanée anglais-français et vice-versa dans de nombreuses réunions au sud du Sahara et le fougueux Teddy (Thadé) Pilley, se faufilant dans la circulation des grandes capitales à scooter avec son «Archie» (le bidule) dans la sacoche.

L'introduction de l'interprétation simultanée dans les cycles d'enseignement de la plupart des Écoles et autres centres de formation de l'après-guerre coïncida en règle générale avec le début du premier cycle d'interprétation. Il n'en fut pas de même à 
l'École d'interprètes de Genève. Son administrateur, le Prof. Antoine Velleman, grand consécutiviste, ne voulut d'abord rien savoir. Ce fut l'Association des diplômés (AIT) qui, encouragée par une lettre envoyée en 1946 de Nuremberg par Marie-France Skuncke, l'introduisit dans ses «soirées de perfectionnement professionnel» qui se tenaient dans le sous-sol de l'église méthodiste de la rue Calvin. Un premier équipement improvisé par Charly Vonwiller en 1947 ne donna pas satisfaction. Nous cherchâmes mieux. L'occasion se présenta au printemps 1949, lors du premier congrès d'après-guerre de l'IMB en Europe à l'hôtel Albert $1^{\text {er }}$ à Bruxelles. Les deux interprètes, Ginette Bazelli (Schaefer) et Wadi Keiser, frais émoulus de l'École de Genève, étaient soigneusement cachés dans un coin de la salle derrière un grand arrangement de fleurs et de buissons alors qu'au centre de la salle, sur une estrade bien en vue de tous, œuvrait M. Graf, le technicien, en combinaison blanche, devant son tableau de bord entouré d'immenses caisses de tubes cathodiques. À la demande des interprètes, M. Graf obtint le prêt à titre gracieux par IBM d'un équipement performant et de deux cabines pour les soirées de formation de l'AIT qui furent opérationnels fin 1949. Peu à peu la direction de l'École se fit à l'idée d'admettre la simultanée dans son curriculum, un professeur (Serge Gloor) vint enseigner au local de la rue Calvin, puis on mit la mention "a suivi un enseignement d'interprétation simultanée » sur le diplôme. Enfin vint la construction de la belle salle d'interprétation simultanée appelée plus tard salle Hélène-Pfaendler - à l'Université même et l'inclusion au plan d'études de cours de simultanée en bonne et due forme sanctionnés par des examens.

\section{Les conditions de travail}

L'étude des conditions de travail des interprètes de conférence pendant la période 1945-1953 est intéressante à deux titres: d'un côté, on constate que des pratiques se mettent en place, notamment à Nuremberg et aux Nations unies, que nous retrouverons sous forme de règles dans les textes de la future Association Internationale des Interprètes de Conférence. Ailleurs, c'est le désert: des interprètes font n'importe quoi sans se rendre compte qu'ils risquent de porter préjudice à leur santé et à la qualité de leur prestation professionnelle.

À Nuremberg, il fallut faire face à deux problèmes découlant du recours à l'interprétation simultanée. D’une part, la fidélité de l'interprétation: l'enjeu des débats était considérable - il y allait de la vie ou de la mort des accusés. D'autre part, la fiabilité du système, étant donné le volume énorme de travail requis sur une aussi longue période.

Dostert avait compris qu'il fallait tout faire pour permettre aux interprètes de bien se préparer aux audiences du tribunal. Il est vrai qu'au début, il fallut vite compléter les équipes - 3 interprètes par langue, quatre langues -, ce qui eut pour conséquence que certains interprètes furent plongés dans le travail «à chaud» presque sans période d'entraînement. Par la suite, pour la plupart des autres, il y eut adaptation graduelle, étude de dossiers, assistance aux audiences en simple auditeur. Écoutons Stefan Priacel, qui n'avait jamais eu de contact avec l'interprétation simultanée avant d'arriver à Nuremberg: "Après un premier essai désastreux que me firent passer Dostert et Jean Meyer, un deuxième essai au cours duquel je prononçais, tant bien que mal, deux ou trois phrases en même temps que l'orateur, leur parut satisfaisant. 
Le colonel Dostert me disait que tout à l'heure mon test avait été très bon. Vous verrez, cela ira très bien. On est comme porté par l'intérêt même qui se dégage de tout ce qui se dit dans ce procès. Le reste n'est qu'affaire d'intelligence, de culture générale, de faculté d'adaptation et de rapidité d'esprit. »

Quant à la charge de travail, les récits de Marie-France Skuncke et Peter Ueberall sont concordants. En tout, pendant le «Main Process», il y avait trois équipes de 16 interprètes qui travaillaient selon un système de roulement astucieux: deux équipes se partageaient la journée, composée de deux audiences de trois heures, la deuxième équipe relayant la première au bout d'une heure et demie. La troisième équipe était libre ce jour-là. Chaque équipe travaillait deux jours de suite, était libre le troisième jour. En séance, le travail se répartissait en fonction des langues parlées en salle. Comme chaque interprète n'assurait normalement l'interprétation que pour une combinaison linguistique, de l'allemand au français par exemple, il arrivait que le travail pendant l'heure et demie en question, était fort mal réparti sur les trois interprètes de la cabine. En effet, certaines interventions pouvaient être fort longues, notamment s'il y avait lecture de déclarations préparées.

L'aspect «préparation continue» était intégré dans le système puisque l'équipe en "stand-by» pendant ses 90 minutes de «non-travail», avait à sa disposition tous les documents de séance nécessaires. Il n’y a aucun doute que ce système de roulement de Nuremberg fut un précurseur du roulement introduit plus tard, bien que selon des modalités différentes, dans le Système de l'ONU et des institutions spécialisées.

À Nuremberg, le travail des interprètes ne se limitait pas à la seule interprétation en séance. Pendant la période de piquet certains interprètes attendaient dans le «Ready Room », d'autres, selon Peter Ueberall, faisaient de la révision des transcriptions des audiences précédentes. En outre, certains interprètes attachés aux délégations nationales avaient aussi pour tâche d'assurer en cas de besoin l'interprétation consécutive des interrogatoires bilatéraux de témoins. À propos de la consécutive, Stefan Priacel note en passant que «chez les interprètes de Nuremberg, exception faite des diplômés de l'École de Genève, le système de prise de notes n'était pas vraiment au point».

Aux Nations unies, fin 1946, le service d'interprétation consécutive comptait 32 titulaires. Vinrent les simultanéistes de Dostert et l'effectif, les deux sections confondues, passa à 78 pour retomber, après la fusion des deux sections en janvier 1948, à 53 interprètes titulaires. Au siège genevois des Nations unies, leur chiffre était resté à 8 . Il va de soi que, pendant les sessions de l'Assemblée, on fit appel à un nombre variable de free-lance.

Pour les interprètes titulaires, intégrés au statut du personnel, les conditions de recrutement et le niveau de rémunération aux Nations unies étaient d'emblée enviables. Thorgevsky rappelle qu'en dehors de leur salaire, "les fonctionnaires “importés" de loin la majorité, s'agissant des interprètes - touchaient une indemnité quotidienne de douze dollars et demi, ce qui sans être fabuleux était tout à fait convenable».

Dès les débuts, les interprètes de l'ONU n'avaient en règle générale, ès qualité, ni heures de bureau, ni obligation de présence et ils ne faisaient que rarement autre chose que l'interprétation. Il arriva qu'on fit appel à ceux qui avaient des langues rares ou des connaissances spécialisées (juridiques, économiques, p. ex.) pour des opérations de 
dépannage urgentes en dehors du service d'interprétation. En revanche, les interprètes momentanément inoccupés organisèrent régulièrement des séances d'entraînement pour parfaire leur connaissance d'une langue supplémentaire.

La spécialisation par matière était difficile à réaliser - il fallait que l'interprète puisse travailler dans tous les domaines -, mais ce qui se produisit assez rapidement, aux Nations unies, ce fut l'instauration d'une sorte d'oligarchie parmi les interprètes. On avait «ses» réunions, le Conseil de sécurité par exemple, et on y restait quelles que fussent les heures de travail, séances de nuit comprises. La «norme Nations unies» (effectif par langue, heures de travail et nombre de séances par semaine) était pour plus tard. Nous y reviendrons.

La spécialisation par langue d'aboutissement, en revanche, s'imposa assez rapidement, surtout après l'avènement de la simultanée. Georges Thorgevsky raconte qu'il n'arrivait que rarement que des interprètes fussent affectés à plus d'une cabine en simultanée. Écoutons-le: «Au cours de la dernière Assemblée générale (1947), Rurik Krymm et Georges Margouliès ont été obligés d'interpréter en simultanée tantôt en russe, tantôt en français, tantôt en anglais; ils l'ont fait avec brio, mais se seraient volontiers passé de cet honneur, car si de telles manœuvres cérébrales sont chose assez courante en consécutive, elles exigent pour la simultanée un effort intense, aboutissant rapidement au surmenage.»

La plupart des organisations intergouvernementales de l'après-guerre immédiat se dotèrent de règles régissant le travail des interprètes s'inspirant du modèle Société des Nations ou de celui de l'ONU. Les grandes conférences diplomatiques firent de même. Mais dans le marché privé les choses allèrent tout autrement.

Dans l'après-guerre, la notion assez floue de «marché privé» ne se fondait pas sur une distinction nette du statut de l'employeur: gouvernemental, intergouvernemental, non gouvernemental, privé à proprement parler. Le marché privé englobait tout ce qui ne relevait pas d'organisations ayant à leur service des interprètes "permanents» intégrés au statut des fonctionnaires et recrutant des free-lance sur une base régulière à des conditions définies d'avance.

Dans «le privé», comme on disait alors, les conditions de rémunération, de travail et de voyage étaient soit dictées unilatéralement par le client, soit négociées entre lui et l'interprète ou le groupement auquel celui-ci appartenait. Les conférences du privé n'étaient pas encore très nombreuses, mais les interprètes de conférence vraiment qualifiés l'étaient encore moins: c'était un «sellers' market, we interpreters being the sellers». Ce facteur, s'ajoutant au prestige des anciens de la Société des Nations et à la renommée des "nouveaux» de Nuremberg et des Nations unies, eurent pour conséquence qu'en règle générale l'interprète de conférence était bien rémunéré et jouissait d'un statut comparable à celui des délégués. Pour la rémunération, les grands consécutivistes essayèrent de se concerter. En fait, ils procédèrent à un calcul à partir de la rémunération mensuelle des interprètes permanents de l'ONU, «fringe benefits» non compris. Ils la divisèrent par vingt jours de travail supposés et arrivèrent à 20 dollars américains par jour (80 francs suisses). Pour tout le reste - effectifs, heures de travail, conditions de voyage -, c'était le flou absolu. "On ne savait pas», nous disent les rares survivants de cette époque.

Il n'y a rien de tel que des exemples vécus pour illustrer l'absence de règles en matière de conditions de travail et de normes techniques à cette époque. L'auteur en cite quelques-uns de son cru, sachant qu'il est loin d'en avoir l'exclusivité. 
- Congrès de l'Internationale socialiste à Vienne, juillet 1948. Les interprètes sont assis au bord du balcon de la grande salle du Parlement de Basse-Autriche. Ils sont reliés au président, seul à disposer d'un micro, les orateurs parlant directement de la salle. Pour les entendre, les interprètes ôtent leurs écouteurs. Heureusement que l'acoustique est excellente et que pour être délégué, à cette époque, il fallait avoir une voix qui porte. Aucun des interprètes n'avait eu de formation en simultanée. Toute l'équipe venait de Genève, par l'intermédiaire de l'AIT (diplômés de l'École). Pour la petite histoire: Vienne était occupée. Dans les rues, les Jeeps patrouillaient avec, à bord, un Américain, un Russe, un Français et un Anglais. Les camarades socialistes autrichiens avaient renoncé à des tickets de rationnement pour nourrir le congrès. Un jeune adjoint du président autrichien s'occupait beaucoup des interprètes: Bruno Kreisky!

- Congrès mondial des intellectuels pour la paix, Wroclav (ex-Breslau), 1950. Langues: anglais, français, allemand, russe, italien, polonais, bulgare, hongrois. Cabines: d'anciennes cabines téléphoniques monoplace, les interprètes assis sur un tabouret de bar, regardant par le petit hublot rond de la cabine sous lequel on avait cloué une tablette de bois. Les seuls interprètes professionnels étaient Richard Herzenberg et Wadi Keiser de Genève. À la séance d'ouverture, au bout de 20 minutes, trois cabines se taisent. On va voir. Les trois collègues s'étaient évanouies. Les cabines étaient pratiquement étanches, mais ces collègues n'avaient pas osé ouvrir les portes à cause des regards courroucés et des «chut» des délégués assis dans le dernier rang à $50 \mathrm{~cm}$ des cabines! Pour la petite histoire: le directeur général de l'UNESCO, Huxley, avait ouvert le congrès, son organisation ayant accepté de parrainer celui-ci. Constatant dès le premier jour que le congrès était totalement infiltré par les communistes, Huxley lui retira son parrainage et repartit immédiatement pour Paris. Mais quelle participation: Picasso, Julie Coton, les Aragon, Paul Robeson, Ilia Ehrenbourg, Fadeyeff, et tant d'autres.

- Congrès de la Fédération internationale des travailleurs des charbonnages, Bournemouth, 1951: Simultanée sans écouteurs ni cabines. Les interprètes étaient installés autour d'une table dans un coin de la salle. 5 langues, 5 interprètes, réunions matin et après-midi du lundi au samedi. Interprètes: Edouard Roditi (F), Laura Sutherland (GB), Maria Roverano (I), Wadi Keiser (D), Juul Poulsen (Scand. et retour en GB). Équipement Simulta. Technicien Dorier.

- Premier Congrès international de médecine homéopathique, Lausanne, 1951. Consécutive debout, anglais, français, allemand. Un interprète. Il s'agissait de résumer en deux fois 5 , maximum 10 minutes, des exposés de vingt à trente minutes. Séances du lundi au samedi, matin et après-midi. Pour la petite histoire: W. Keiser s'effondra à la fin de la séance de clôture, fut remis sur pied par le Dr Pahud, président du Congrès, dans sa clinique homéopathique. Prix de consolation: un bel ouvrage sur l'homéopathie avec dédicace du président.

Les trois premiers congrès cités avaient ceci en commun: le soir, après les séances, on restait sur place pour traduire les projets de résolution, les décisions arrêtées, certains discours importants qu'il fallait distribuer en séance le lendemain. Cela faisait tout normalement partie du travail. Parfois, malgré notre jeunesse, nous nous rendions compte que nous étions à la limite. Alors nous allions nous défouler. $\mathrm{Au}$ Congrès de Wroclav, ville détruite à $80 \%$, il y avait une exposition pour les «terres récupérées» avec un Lunapark. Alors, après les réunions, nous allions au cassecroûte: saucisses, bière et tout, mais surtout, nous allions nous défouler sur les carrousels, montagnes russes et autres stands de tir. Puis, vers 21 heures, retour à nos traductions, parfois jusqu'à une heure du matin.

Une autre tâche attendait l'interprète auprès de certains employeurs, notamment, mais pas exclusivement, en République fédérale d'Allemagne: la rédaction des 
«notes de séance», (Aufzeichnungen). Lors d'entretiens entre hommes d'État en tête-à-tête, l'interprète étant la seule autre personne présente, celui-ci était chargé de rédiger un compte rendu sommaire à remettre à, mettons, M. Adenauer ou M. Hallstein. Cette rédaction se faisait immédiatement après l'évènement. En Allemagne, il était entendu que ce texte n'engagerait pas la responsabilité de l'interprète: c'était un simple aide-mémoire, mais cela impliquait une prise de notes différente de celle d'une consécutive ordinaire. Erich Feldweg nous raconte que ce genre de travail pouvait prendre des proportions considérables. Ainsi, pour le chancelier fédéral Kurt Georg Kiesinger, il dicta en quelques semaines environ 130 pages de «Aufzeichnungen », en général en dehors des heures de service. En outre, pendant longtemps, cette activité se fit "en cachette», parce que l'autre côté était sensé croire qu'il n'y avait pas de trace écrite de l'entretien. Erich Feldweg nous dit que ce tabou ne fut rompu qu'en 1972 lorsque, pour la première fois, les médias parlèrent de l'existence de ces «Dolmetscher-Aufzeichnungen».

Les réunions syndicales étaient de loin les plus exigeantes au point de vue des activités s'ajoutant à l'interprétation en séance. Pour les syndicalistes, l'interprète faisait partie de la famille et il était sensé se tenir à disposition du lever au coucher : tout le monde logeait au même hôtel, mangeait aux mêmes restaurants et la plupart des délégués dépendaient totalement des interprètes pour s'entretenir avec les camarades parlant d'autres langues.

À cette époque, il était tout à fait normal d'exiger des interprètes qu'ils travaillent pendant les excursions touristiques, assis, par exemple, à l'avant de l'autocar à côté du guide, décrivant en trois langues les beautés du paysage.

Toujours à propos d'activités autres que l'interprétation. Au début des années 1950, E. Glenn, chef de la section interprétation du Service linguistique du State Department des États-Unis, eut une idée de génie: Pourquoi ne demanderait-on pas aux interprètes, rompus aux astuces de la procédure des réunions internationales, d'assurer la présidence de telles réunions? Pourquoi ne pas en faire des experts qui, tour à tour, serviraient la communication en interprétant pour telle réunion, en présidant telle autre? Hélas! ce projet fascinant fit long feu.

Une chose aura sans doute frappé les lecteurs: Comment des interprètes pouvaientils travailler seuls en simultanée des journées entières? Il faut savoir qu’à cette époque, sauf pour les échanges techniques courts demandant une précision absolue, l'interprétation simultanée devenait souvent, au bout d'une heure ou plus, une sorte de «running commentary» qui restait tout à fait cohérent et permettait aux participants de suivre et d'intervenir s'ils souhaitaient qu'on revienne plus en détail sur tel ou tel point. Cela dit, l'endurance des interprètes de ces temps-là était plutôt remarquable. Preuve en est ce témoignage d'Elisabeth Meister: "C'était en 1948, à la Conférence européenne de radiodiffusion au Danemark; il s'agissait de réorganiser les services de diffusion après les destructions de la guerre. Les Soviétiques font obstruction dès le début de la conférence sur des questions de procédure, impossible de passer au fond. À la troisième semaine, le président décide de ne pas lever la séance avant que ces questions ne soient tranchées, autrement dit, il veut avoir les Soviétiques à la fatigue. Je suis en cabine française dès 9 heures du matin avec un collègue qui ne donne pas satisfaction et on me fait savoir que je continue seule. Pour le russe, je prends le relais sur la cabine anglaise où il y a deux interprètes. La séance dure toute la journée, à 
midi et à 19 heures on m'apporte des sandwichs en cabine. Les délégués sont à la même enseigne. La séance se poursuit jusqu'aux petites heures du matin suivant, j'aurai été 16 heures d'affilée en cabine, seule. Mais les Soviétiques ont cédé et la conférence peut démarrer...». Elisabeth raconte qu'à son retour de la conférence, son médecin diagnostiqua une primo-infection et lui conseilla trois mois de repos complet. Réponse d'Elisabeth: «Impossible, je repars la semaine suivante pour mon prochain engagement.»

Ce cas extrême illustre bien la remarque citée plus haut: «On ne savait pas...» Ce ne fut qu'une dizaine d'années plus tard qu'on commença sérieusement à se poser des questions sur la santé des interprètes. Jean Herbert, dans un article paru dans L'Interprète en 1956, fut le premier à lancer le débat. Cet article suscita une réponse d'Elisabeth Meister et un article de Wadi Keiser: "Problèmes de santé dans l'interprétation de conférence", repris à la même époque par les interprètes fonctionnaires de l'OTAN pour leur dossier. Ceux-ci furent, à notre connaissance, les premiers à se lancer dans une revendication pour une charge de travail normale.

À propos de revendications, il est intéressant de noter que les conditions de travail décrites ci-dessus ne furent guère contestées jusqu'au début des années 1960; les actions menées par les interprètes ou leurs associations se limitant jusque là à des revendications tarifaires. Le changement n'intervint sans doute qu'au moment où la pression de la charge de travail devint constante pour les permanents alors que le feu sacré des années d'édification de leur organisation cédait au souci de préserver la santé et la qualité du travail. Baigorri-Jalón a trouvé une excellente formule en disant qu'aux Nations unies, l'arrivée de la génération intermédiaire d'interprètes au début des années 1960 «a signifié le passage de l'interprétation vue comme prodige à l'interprétation vue comme une profession». Cela vaut également pour les Communautés européennes où les années euphoriques de la mise en place des institutions et la multiplication des sessions-marathon débouchèrent sur cinq années de lutte acharnée pour l'amélioration des conditions de travail: présentation du «cahier des doléances» en 1963, aboutissement de l'action en 1967. Aux Nations unies, le point culminant de cette action fut marqué par la grève des interprètes de 1974. Plus loin dans l'ouvrage, nous évoquerons l'action de l'AIIC en matière de rémunération et de conditions de travail des interprètes free-lance travaillant pour les trois grandes familles d'organisations intergouvernementales (ONU, CEE, Coordonnées), conduisant en 1969 à la conclusion des accords quinquennaux. Nous évoquerons aussi l'héroïque «bataille du Berlaymont» à Bruxelles à la même époque, action conjointe des interprètes fonctionnaires de la Commission CE et de l'AIIC.

\section{La formation}

Dans l'après-guerre immédiat et pendant une bonne dizaine d'années, les interprètes issus d'une école d'interprétation étaient en minorité. Mais la forte demande en interprètes qualifiés dès la fin des hostilités entraîna la création d'un nombre considérable d'écoles d'interprétation et autres centres de formation. Après l'École d'interprètes de l'Université de Genève (1941), il y eut Heidelberg - ressuscitée - et Germersheim (1946), Paris HEC (1948), Vienne et Munich (1952), Paris ESIT (1957). Un chapitre spécial de cet ouvrage sera consacré à la politique de l'AIIC en 
matière de formation et de collaboration avec les écoles d'interprétation. Quelques réalisations et un litige méritent cependant d'être signalés pour la période pré-AIIC et le début de l'Association.

Les premières écoles du type universitaire se caractérisaient presque toutes par des programmes complets de traduction-interprétation s'échelonnant sur plusieurs années, le cycle interprétation se situant au niveau de la licence universitaire voire d'un titre post-universitaire. Très tôt, cependant, d'autres cycles de formation furent conçus et firent preuve d'efficacité, s'imposant même dans certains circuits: la formation courte, la formation hors école par des employeurs institutionnels, l'organisation par les écoles de cours spéciaux hors curriculum à la demande d'employeurs ou gouvernements. Nous avons déjà parlé de la formation des interprètes pour les missions de productivité par le State Department. Voici encore trois exemples:

- Le «Interpreters' Working Party» de Londres. A.T. Pilley eut un coup de génie en ressuscitant en 1946 le "Linguist's Club», logé dans une maison de 5 étages du côté de Grosvenor Place, offrant aux jeunes contre une «fee» modique des cours de langue, danse, ping-pong, échecs, une bibliothèque, une cantine et, aux futurs interprètes, son «Working Party». Ce cours se distinguait par un minimum de «frills», une grande souplesse, une bonne sélection des candidats (test d'aptitude éliminatoire), une formation collant à la réalité professionnelle et, pour ceux qui réussissaient, une aide puissante au démarrage professionnel. La durée des «études» dépendait du talent et de la diligence des participants. Le nombre impressionnant d'interprètes qualifiés sortis de ce cours prouvait qu'il était possible de former de très bons interprètes en très peu de temps. Pour la petite histoire: le Linguist's Club était, pendant des années, le lieu de rencontre de presque tous les interprètes continentaux passant par Londres. Ainsi, pendant la Conférence pour le règlement des dettes extérieures allemandes qui se tenait à Lancaster House et s'échelonna sur plus d'une année (1950-1951), le Linguist's Club fut l'endroit idéal pour se détendre après le travail. Toujours pour la petite histoire: à la Conférence des dettes allemandes, le secret professionnel des interprètes fut mis à rude épreuve. Sur une période de six mois, les interprètes travaillant aux réunions «top secret» du «Steering Committee» furent mis trois fois sur le gril par Scotland Yard. Le lendemain de certaines réunions du Comité de direction un compte rendu presque complet paraissait dans le Financial Times avec les mouvements en bourse immédiats qu'on devine. On soupçonna les interprètes, seuls présents en plus des chefs de délégation et de leurs adjoints. Enfin le coupable fut démasqué: un des adjoints du chef de la délégation britannique! Mais revenons à la méthode du «Working Party». Teddy Pilley l'appliqua avec succès pour la formation rapide des équipes d'interprètes de certains grands congrès d'abord - p. ex. le Congrès sioniste à Jérusalem en 1952, quatre langues, 11 interprètes -, pour le recrutement et la formation d'interprètes permanents de deux parlements du Common wealth britannique ensuite: le House of Representatives à Colombo en 1955 (anglais/tamoul et cinghalais) et l'Assemblée législative à Singapour en 1957 (anglais/tamoul/malais et mandarin).

- La formation par les employeurs. Le premier projet de ce genre fut le projet No 198 de l'Agence Européenne de Productivité annoncé le 12 décembre 1953 sous le titre «Sélection et formation d'interprètes simultanés ». L'AEP, une agence de l'Organisation européenne de coopération économique, avait rencontré des difficultés à recruter des interprètes qualifiés en nombre suffisant pour ses nombreuses missions et réunions de productivité qui avaient lieu dans tous les coins de l'Europe. Le but était de créer un centre de formation au service des pays participants. Deux idées maîtresses: A) Assistance professionnelle et technique à l'échelon national pour les réunions sur place dans les pays participants portant sur la présélection de candidats, les examens d'aptitude 
professionnelle, la formation et le perfectionnement professionnel. B) Pour les conférences européennes, création d'un pool européen d'interprètes permanents (présélection, formation, etc.). Il ressort d'un rapport rédigé après une réunion d'experts par W. Keiser, membre de la délégation suisse, que 8 pays étaient intéressés: Allemagne, Belgique, Danemark, France, Italie, Suisse, Turquie, États-Unis d'Amérique (plus l'OECE et l'AEP). En décembre 1954, le projet fut abandonné, essentiellement à cause de la crainte qu'il pourrait porter préjudice au pool des interprètes fonctionnaires de l'OECE et de la peur des écoles d'interprétation de certains pays d'être court-circuitées. Bien que ce projet débouchât sur un échec, la formule fut reprise dans son principe par quelques employeurs institutionnels.

- Cours ad hoc organisés par une école à la demande d'un employeur. En 1953, M. A. Spira, chef de la section interprétation de la Haute Autorité de la Communauté européenne du charbon et de l'acier (CECA) chargea l'École de Genève de l'organisation d'un stage de six semaines de formation accélérée ou de perfectionnement pour ses interprètes. Un «crash course» fut organisé, l'École faisant appel aux meilleurs de ses enseignants et à quelques interprètes professionnels extérieurs. Ce fut un succès et cette expérience servit de modèle à des cours semblables ultérieurs, à Genève, à Paris (ESIT) et ailleurs. En marge : à ce stage nous fîmes la connaissance d'une philologue trilingue charmante, Renée Herz qui, devenue Renée van Hoof, accéda par la suite à la direction de ce qui allait devenir le plus grand service d'interprétation du monde, celui de la Commission de la Communauté économique européenne à Bruxelles.

Le litige. Curieusement, le premier litige judiciaire opposant une association professionnelle d'interprètes à une tierce partie se joua sur l'arrière-plan de la formation. En décembre 1954, répondant à un appel au secours d'un groupement de collègues d'Italie représenté par Guido Enk et Fred Müller, le président de l'AIT, agissant au nom du comité de l'Association, envoya une circulaire (sic!) aux membres, avec copie à l'AIIC, la FLIG et LACI, leur demandant de ne pas accepter une éventuelle offre pour le Congrès mondial du pétrole à Rome en 1955 qui leur serait adressée par «M. Baridon ou ses émules », même si "pour une fois on leur offrirait le plein tarif». Ce fut «l'affaire Baridon»: plainte pénale en diffamation et concurrence déloyale contre l'AIT et son président devant le tribunal de Lugano; innombrables tractations, interrogatoires, frais importants. Les deux parties ayant choisi des avocats connus pour être des "étouffeurs de procès", tout se termina en mai 1957 par un arbitrage débouchant sur un acte de conciliation (arbitre conciliateur: Alvise Savorgnan di Brazzà; témoins: Jean Herbert, Maria Grazia Riontino, directrice du Centro Congressi di Milano; signataires: W. Keiser, président de l'AIT et le Prof. Silvio Baridon, directeur des Écoles de Milan, Florence et Rome). M. Baridon était le vainqueur, sur papier, son but ayant été de se faire connaître, d'étendre sa chaîne d'écoles ailleurs en Europe, accessoirement de faciliter l'admission de ses diplômés à l'AIIC et de créer une position de force à son Centro Congressi. De fait, en dehors de l'Italie, les fruits escomptés ne tombèrent pas dans l'escarcelle de M. Baridon : l'école d'interprétariat fondée par lui à Paris fut bien vite éliminée, essentiellement grâce à Zoran Seleskovitch, et remplacée par une véritable école d'interprètes de conférence, devenue l'ESIT en 1957. Aucune faveur ne fut accordée par l'AIIC aux diplômés des écoles italiennes de Baridon. En Italie, en revanche, surtout à Milan, l'AIIC et ses membres souffrirent énormément. Le marché italien étant inondé des diplômés de ces écoles, la règle du contrat direct n'étant pas acceptée par le Centro Congressi de Milan, d'autres OPC suivant l'exemple de celui-ci, nos collègues d'Italie membres de l'AIIC 
perdirent des centaines de journées de travail. Avec le recul historique, on peut se demander si une politique de plus grande souplesse en matière d'admission de la part de l'AIIC - à l'instar de celle pratiquée à d'autres moments dans d'autres régions - et une opération de diplomatie vis-à-vis du Centro Congressi aurait peut-être permis à l'AIIC de s'implanter plus facilement dans cette région où tout était encore en devenir et à nos collègues, dont le courage était admirable, de moins souffrir.

À propos de litiges: par la suite, l'AIIC elle-même allait être entraînée dans des litiges. Nous en parlerons sans doute plus tard, mais il est intéressant de noter que chaque fois, il y allait de principes fondamentaux de notre profession. Lors de «l'Affaire américaine»(1967), il s'agissait du principe de l'universalité de l'AIIC et de la libre circulation des interprètes de conférence, dans «l'affaire Falkenburger» (1972) du contrat direct, dans les trois autres cas - Canada (1982), Allemagne (Kartellamt, 1986), États-Unis (FTC, 1993-98) - de toutes les règles et normes visées par le droit de la concurrence.

\section{Dispersion géographique, circuits, marchés}

Sous ce titre nous ne reparlons que brièvement des grandes institutions intergouvernementales onusiennes ou européennes. Signalons cependant que la thèse de Baigorri-Jalón est une mine d'or pour ce qui est des Nations unies. D’autres circuits ou marchés de l'interprétation se développèrent. Genève, Paris, Londres et New York perdirent une partie de leur prédominance et la demande sans cesse croissante en interprètes devint de plus en plus polycentrée et diversifiée.

En règle générale, les grandes organisations internationales se dotèrent de services de traduction et d'interprétation employant des fonctionnaires, "les permanents », optant selon les besoins pour des unités à effectifs assez nombreux ou pour de petits noyaux de permanents - ainsi pendant tout un temps Ludovic Ravet, Hans Jacob, Marie-France Skuncke, Guy Piquemal et Patricia Longley furent les seuls permanents de l'UNESCO -, mais toutes avaient besoin de recourir à des interprètes indépendants, les free-lance, pendant leurs périodes de haute activité. Très vite, ces organisations eurent «leurs» free-lance réguliers, recrutés par priorité sur les autres, dans certains cas sur la base de contrats cadre. Zoran Seleskovitch, visionnaire, disait qu'en fait, ces free-lance allaient devenir des "permanents au rabais », assurant parfois un nombre de jours de travail mensuel supérieur à celui de leurs camarades permanents, sans la sécurité d'emploi et les «fringe benefits » de ces derniers et qu'il fallait au plus vite négocier des conventions collectives. Christopher Thiéry nous rappelle que Zoran était particulièrement soucieux de la responsabilité des organisations en matière de retraite et de sécurité sociale des free-lance - idée qui était venue à fort peu de gens à l'époque. Ce fut le moteur essentiel des négociations pour les accords - la rémunération et l'indexation venant pratiquement en sus. Mais pour cela, il fallait d'abord créer une organisation professionnelle reconnue comme interlocuteur valable par ces organisations.

Pour le secteur non-gouvernemental, nous nous limiterons par la force des choses à la description de quelques exemples caractéristiques tout en invitant nos lecteurs à apporter les compléments d'information susceptibles de faire l'objet d'une publication ultérieure sous forme de monographies historiques par secteurs, pays ou régions. 
Très tôt, deux circuits importants se mirent en place, celui des organisations syndicales et celui des congrès médicaux. Là aussi, il y avait les habitués pour ne pas dire les spécialistes. En effet, ces organisations aimaient s'assurer le concours d'équipes d'interprètes fidèles qui les suivaient partout dans le monde, quitte à compléter l'équipe de base d'interprètes locaux, notamment pour des langues autres que les langues officielles de l'organisation en question. Très vite aussi, certains de ces interprètes spécialisés devinrent interprètes-recruteurs, "consultant interpreters», tels que Alfred Spirig et Karl Gärtner dans le secteur syndical où ils obtinrent auprès de plusieurs secrétariats professionnels internationaux (SPI) de notables changements pour le mieux en matière de conditions de travail, ou George Gérard, René Pinhas, Peter Spitz et Jean Meyer pour les congrès médicaux. Ce dernier, d'ailleurs, avait réussi à se placer à la source en tant qu'interprète-conseil du Comité international des organisations scientifiques (avant tout médical) à Paris. Particularité des engagements offerts par Meyer: jamais de contrat, cela se limitait à un accord sur la disponibilité, on se trouvait sur place au moment convenu et Jean vous glissait au début de la conférence une enveloppe contenant le montant total en espèces, frais de voyage compris. Marie Ginsberg était autant active dans le circuit syndical que dans celui des réunions médicales. De même pour Charly Vonwiller qui devint par la suite chefinterprète de la Fédération syndicale mondiale à Vienne, qu'il quitta lorsqu'elle déménagea à Prague.

C'est dans ces réunions que nous rencontrâmes les premiers interprètes de conférence des pays excentriques par rapport aux principaux centres d'activité internationale, en Scandinavie, par exemple. Il y avait là le mélomane Josef Berg à Oslo, le Danois Juul Poulsen à Copenhague, le légendaire Suédois Sven Backlund qui, syndicaliste convaincu de la supériorité du "modèle scandinave», décidait souverainement ce qui était susceptible d'intéresser «ses délégués », donc digne d'être interprété et remplissait le reste du temps par des renseignements touristiques ou les dernières blagues. Par eux, nous fîmes la connaissance des autres collègues scandinaves, tels que Ellen Larsen, Axel Anslev, Hannelore Aaberg et Jeannette Vinkel-Dartnell à Copenhague, Margot Levy et Calman de Pandy, historien à ses heures, à Stockholm. D'autres lumières à l'horizon syndicaliste de cette époque furent Erich Wächtler et l'incollable Elfriede Tschiesche à Vienne et l'original Gérard John à Genève, syndicaliste dans l'âme, longtemps président de l'AIT et profondément convaincu de la règle de la disponibilité de l'interprète 24 heures sur 24.

Dans le domaine médical aussi, les interprètes «spécialisés» se rencontrèrent régulièrement. Spécialisés, soit parce que médecins eux-mêmes, tels que Daar, Destouches, Missirliu, Licea Romei, Valenzuela plus tard, soit à force de s'y être attelés avec passion, comme Behringer, Meister, Lambert, Pfaendler, Simha, Schaefer, TestotFerry, De Clarens, Apaire, Pinhas, Spitz, Thiéry, Band, Schmidt pour n'en mentionner que quelques-uns. Il y avait aussi, parfois, des équipes mixtes. Un seul exemple: en 1952 se tint à Copenhague le Congrès mondial de la poliomyélite, au moment même de la dernière grande épidémie de cette terrible maladie en Scandinavie. Les interprètes des cabines anglaise et française étaient des habitués des équipes de George Gérard. Pour le complément allemand, les organisateurs firent appel à l'École de Genève et trois membres de l'AIT furent spécialement formés sur une période de quatre mois par des médecins de l'Hôpital cantonal de Genève. Au Congrès, tout se passa bien, sauf que, dans la session de chirurgie reconstructrice, les écrans étant à 
cinq mètres des interprètes, la cabine allemande soudain se tut: les deux jeunes collègues n'avaient jamais vu une opération sanglante et elles étaient tombées dans les pommes! Pour l'histoire: c'est à ce congrès que deux médecins américains, les docteurs Salk et Sabin, annoncèrent leurs premiers résultats cliniques prometteurs avec un vaccin anti-polio, le premier sur base d'un virus mort, le second avec un virus vivant atténué. Finalement, ce fut le Dr Salk qui emporta la course en présentant ses résultats concluants au congrès suivant, à Rome, trois ans plus tard.

La dispersion géographique des interprètes variait de pays en pays. Alors qu'en Suisse la très grande majorité des professionnels de l'interprétation de conférence habitait à Genève, avec un petit noyau à Zürich autour du groupement des anciens de l'École de Genève dirigé par Iris Vonow, et qu'en France en dehors de Paris et de Strasbourg il n'y avait presque rien, dans d'autres pays, l'Allemagne et l'Italie par exemple, le polycentrisme fut la règle dès le début. Prenons un seul exemple, l'Allemagne: Le service linguistique du ministère des Affaires étrangères de la RFA, dirigé par le Dr Reichhold, avait pour chef-interprète Heinz Noack. Les taux pratiqués par le «Sprachendienst» servirent en gros de taux indicateurs pour les interprètes recruteurs dans le reste du pays. Bonn attira de nombreux interprètes étrangers, dont certains furent engagés comme fonctionnaires (Colette Bouverat, Heidi Straub-Weber, Madeleine Picard, par exemple) à côté de leurs collègues allemands, dont Heinz Weber qui succéda à Noack et resta chef-interprète jusqu'à sa retraite en 1989. Noack devint, en 1953, chef du service de traduction de la Haute Autorité de la Communauté européenne du charbon et de l'acier à Luxembourg. Puis il y eut les interprètes-recruteurs, les «market-makers", dans plusieurs grandes villes allemandes: Reinhard Lochner à Hambourg, futur président de l'AIIC, Walter Jumpelt à Bonn, plus tard grand animateur de la Commission technique de l'AIIC, Helga Brunner à Düsseldorf, les Klitscher et Erich Feldweg à Munich, sans oublier Günther Haensch qui allait devenir chef-interprète à la CECA à Luxembourg, co-directeur de l'École de Munich (fondée par Paul Schmidt) et auteur-éditeur de plusieurs ouvrages de terminologie, et surtout, à Berlin, Donata Helmrich, la doyenne de l'interprétation de conférence. Ces collègues recruteurs s'adressaient autant aux quelques free-lance allemands de l'époque, les Alenfeld, Longerich, Klebes, Kuhn, Quambusch, Freichels, von Seemann, Frisch, Merten, qu'à des free-lance venant des pays voisins. Hermann Kusterer, fonctionnaire du Auswärtiges Amt depuis 1951, avait été détaché à la CED et à l'OTAN à Paris pour revenir en 1957 à Bonn au Sprachendienst dont il allait devenir le chef en 1971.

En dehors de l'Europe, abstraction faite des pôles des Nations unies à New York et Washington et du State Department américain, il y avait de petits groupes d'interprètes, voire parfois un seul, qui étaient en liaison avec leurs collègues européens et allaient souvent devenir les relais pour l'extension de l'AIIC au reste du monde. Pour l'hémisphère ouest, mentionnons d'abord au Canada Thérèse Romer, mais aussi Noël Salathé et Fernand Cordier de l'OACI. Aux États-Unis ce furent Vladimir Pojidaeff, Henry Lunau, Marc Moyens, Fernando Morales Macedo, Ingeborg Wichmann, Lisa Valyiova, Margarethe (Maxie) Brooks (Bowen), Harris Coulter, Stefan Horn à l'École de Georgetown, Claude Badoux (Intelsat/Comsat), Edmund Glenn au State Department et d'autres qui marquèrent la scène de l'interprétation. Grâce à eux et aux liens qui s'étaient tissés entre l'Europe et les États-Unis, notamment à l'occasion des missions de productivité, la fondation presque conjointe de l'AIIC (1953) et de la 
TAALS (The American Association of Language Specialists, 1955) et leur extension aux Amériques allait pouvoir se faire presque sans heurts. La double appartenance de nombreux interprètes aux deux associations et la quasi-identité de leurs règles fondamentales en furent les garants et allèrent en assurer la survie lors de la triste «affaire américaine» dont il sera question plus loin.

Dans certains pays, l'implantation de l'interprétation de conférence fut marquée par des maîtresses-femmes qui allaient jouer un rôle décisif dans l'extension de l'AIIC: Italia Moraita au Mexique, entourée des fidèles Heberto Sein et Guido Gomes de Siva. Edith van de Beuque au Brésil, qui allait se fâcher avec l'AIIC après l'avoir introduite à Rio de Janeiro, Sergio de Albuquerque et Dorothée Kogut reprenant le flambeau en attendant la constitution du groupe très actif de Saõ Paulo. Anne Robson en Australie, longtemps seul membre de l'AIIC dans ce vaste pays, Marcela de Juan en Espagne, entourée de Paco Caballero, Teresa Oyarzun, Elsa Yndurain et quelques autres. Mentionnons encore, pour l'Amérique latine, Anne Randle, Ruth James, Hector de Urquiaga, Emilio Stevanovitch qui resta en dehors de l'AIIC tout comme d'autres interprètes, pourtant excellents, notamment parmi les permanents.

Deux parties du monde allaient rester longtemps réfractaires à la pénétration par l'AIIC, les pays du bloc communiste, d'une part, le Japon d'autre part. Dans les deux cas, la quasi-impossibilité pour les interprètes de conférence d'adhérer à l'AIIC était inhérente au système prévalant bien avant la création de l'Association. Les régimes communistes interdisaient l'affiliation individuelle de leurs citoyens à des organisations professionnelles étrangères. De plus, une des règles les plus fondamentales de l'AIIC, celle du secret professionnel absolu, était inapplicable puisque les mandants des interprètes exigeaient d'eux de rapporter tout ce qu'ils entendaient dans l'exercice de leur profession et en dehors.

$\mathrm{Au}$ Japon ce fut le système hiérarchique traditionnel qui fit obstacle à certaines règles fondamentales de l'AIIC, par exemple celle du contrat direct et celle de la même rémunération pour tous les membres d'une même équipe. Cela découragea les interprètes japonais d'adhérer à l'AIIC du moment qu'ils faisaient pratiquement tous partie d'une entreprise fortement hiérarchisée ou dépendaient d'un PCO qui ne voulait pas entendre parler de telles hérésies. Cela n'empêcha cependant pas l'établissement d'excellents liens de coopération et d'amitié entre certains collègues recruteurs des États-Unis et d'Europe et les pionniers de l'interprétation de conférence au Japon que furent Musami Muramatsu fondateur de SIMUL International, Tatsuya Komatsu, son lieutenant et directeur de la SIMUL Academy ou, sur un autre plan, Mme Saito, directrice de l'école d'interprètes de l'Université catholique de Tokyo. Ce ne fut que plus tard que des interprètes de langue japonaise, recrutés au Japon ou à l'étranger, allaient être acquis à la cause de l'AIIC grâce à l'exemple d'indépendance qui leur fut donné par des collègues étrangers venus travailler au Japon et grâce à l'introduction de la langue japonaise dans certaines organisations internationales, notamment le Bureau international du travail. Deux interprètes firent ouvre de pionniers à cet égard, Ken Yokota à Tokyo et Reiko Blauenstein-Matsuba à Genève.

Vers la fin des années 1940 et au début des années 1950, certains développements commencèrent à préoccuper des interprètes soucieux du statut de la profession, tant permanents que free-lance. D'un côté, il y eut la formation d'associations de traducteurs et d'interprètes, parfois attachées à une école, parfois carrément conçues sous forme de groupements nationaux englobant toutes les catégories professionnelles de 
la branche. Mentionnons, à titre d'exemple, Universitas (diplômés de l'École de Vienne), la Société française de traducteurs, la Chambre belge des traducteurs, philologues, et interprètes, la Translatörforeningen du Danemark, puis, plus tard le Bund deutscher Dolmetscher und Uebersetzer en Allemagne, l'Associazione Italiana dei Traduttori ed Interpreti en Italie. N'oublions pas qu'à cette époque, les pays et les professions étaient encore très marqués par le souci de se reconstruire et de s'organiser dans une ambiance et aspiration nationalistes. Le risque pour les interprètes de conférence était évident de voir se créer des cloisonnements nationaux, des barrières au libre exercice de cette profession internationale par définition.

De leur côté, les grands employeurs internationaux d'interprètes commencèrent aussi à se cordonner ou se concerter, par exemple, aux Nations unies, par le Comité consultatif pour les questions administratives (CCQA). Les grands employeurs devenaient de plus en plus puissants et on pouvait craindre qu'ils finissent par imposer leurs conditions aux interprètes. Il fallait réagir.

\section{Les associations et groupements d'interprètes de conférence précurseurs de l'AIIC ou contemporains (1945-1957)}

Réagir, avons-nous dit. En d'autres termes organiser la profession. Pourtant, cela n'alla pas tout seul. En effet, la majorité des interprètes de cette époque étaient des personnalités souvent brillantes, fort remuantes et surtout de farouches individualistes. Il ne fallait pas leur parler d'un syndicat.

Les premières initiatives d'organisation professionnelle partirent soit d'associations d'anciens étudiants, soit de petits groupes d'interprètes indépendants travaillant régulièrement pour les mêmes organisations internationales ou ministères nationaux et de quelques interprètes fonctionnaires de ces organisations. Selon Denis Cru, un éphémère Institut international des interprètes (III) fut fondé en 1946 par quelques interprètes permanents des Nations unies à Lake Success (président Jean Herbert, trésorier Georges Kaminker), mais on n'en sait rien de plus.

Les premiers à s'organiser sérieusement furent les diplômés de l'École d'interprètes de l'Université de Genève. Leur Association des anciens élèves (AAEEI, plus tard rebaptisée Association d'interprètes et de traducteurs - AIT) fut fondée en octobre 1945. Fin 1946, elle compta 123 membres répartis dans 11 pays. Véra Wegelin fut la première présidente, Iris Vonow la principale animatrice de la section de Zürich. Le Bulletin de l'Association (novembre 1945) prit le nom L'Interprète dès le deuxième numéro et fut le premier journal professionnel imprimé paraissant régulièrement et mettant spécifiquement l'accent sur l'interprétation de conférence. Il resta le seul du genre jusqu'à la sortie du premier Bulletin imprimé de l'AIIC en janvier 1973. Ses articles de fond sur les problèmes professionnels et sur les décisions des assemblées de l'AIIC firent date.

L'AIT s'était dotée d'un bureau de placement, mais se ravisa rapidement lorsque les autorités genevoises l'informèrent des formalités à remplir et des conséquences fiscales. Désormais elle se bornait à répartir le travail parmi ses membres et publiait dès 1947 un annuaire des membres traducteurs et interprètes et un tarif en bonne et due forme. En voici un extrait: 
Afin d'assurer la protection des intérêts professionnels des membres de l'Association, l'Assemblée générale décide d'établir des taux minima de rémunération pour les travaux d'interprétation et de traduction.

Les membres de l'A.A.E.E.I. sont invités dans l'intérêt commun, à ne pas accepter de rémunération inférieure à ce minimum; ils sont cependant libres de travailler à titre gracieux, si, pour des raisons personnelles, ils désirent le faire.

Taux minima de rémunération

\section{Honoraires}

1. Interprètes aux conférences internationales: Fr. 80. - par jour et rémunération supplémentaire à partir de la troisième langue: Fr. 20. - par langue

2. Interprètes stagiaires: Fr. 50. - par jour (possibilité de fractions horaires pour cette catégorie seulement)

3. Interprètes d'affaires: Fr. 50. - par jour, ou, en cas d'engagement prolongé, forfait.

4. Traducteurs aux conférences internationales: Fr. 80. - par jour et rémunération supplémentaire à partir de la troisième langue: Fr. 20. - par langue

Frais de voyage

Les frais de voyage sont à charge de l'employeur: il y a lieu de prévoir le déplacement par avion ou chemin de fer $1^{\text {ere }}$ classe (wagon lit) pour les voyages hors du pays de résidence.

Les honoraires et frais de déplacement sont dus à partir du jour de départ au jour de retour, y compris les jours fériés.

On notera les différences de ce système par rapport au futur tarif de l'AIIC: supplément pour langues supplémentaires à partir de la troisième langue; tarif pour débutant (stagiaires), un point qui fera l'objet d'un grand débat de doctrine peu après la fondation de l'AIIC; voyage en première classe, avion comme train; honoraires complets pendant toute la durée d'absence, jours fériés compris. Quant aux «frais de déplacement» (per diem), ils ont fait l'objet d'une première concertation entre associations internationales. Nous y reviendrons lorsqu'il sera question de la IACIT.

L'AIT eut un centre d'entraînement professionnel dès sa fondation. Nous en avons parlé sous «formation» ci-dessus. Elle déployait son activité d'organisation d'équipes d'interprètes dans toute l'Europe et avait des liens de coopération et d'échange de publications avec plusieurs associations d'anciens étudiants d'autres écoles ou avec des associations de traducteurs et interprètes nationales en France, Allemagne, Pays-Bas, Danemark, Belgique, Autriche, Italie. L'auteur se souvient d'une rencontre avec une charmante représentante d'Universitas au café Sacher à Vienne: Dipl. Dolm. Dr Margarethe Brosch (Maxi Bowen). Nous convînmes entre autres d'un échange "Mitteilungsblatt» contre L'Interprète.

La plupart de ces associations étaient orientées sur la défense des professions de traducteur, accessoirement d'interprète, dans leur cadre national. Par la suite, elles devinrent presque toutes membres de la Fédération internationale des traducteurs. Les seules autres associations déployant alors une activité spécifique pour les interprètes de conférence étaient la IACIT (International Association of Conference Interpreters and Translators, Londres), la FLIG (Free Lance Interpreters Geneva) et, avec centrage sur le Royaume-Uni, la LACI (London Association of Conference Interpreters).

Avant de parler des trois associations, il faut évoquer le mystère de l'AMICI (Association mondiale des interprètes de conférences internationales). Nous avons trouvé 
des références à un tel groupement dans des procès-verbaux de réunions du comité de l'AIT, au début des années 1950. Il aurait été basé à Paris. Il se serait agi d'une association destinée à prendre la succession de la IACIT, en perte de vitesse. En fait, l'AMICI n'a jamais existé. C'est Roger Glémet qui dissipe le mystère: «Oui, c'est bien moi qui, en août 1950 à Strasbourg, au Conseil de l'Europe, ai proposé la création d'une association mondiale des interprètes de conférences internationales. » André Kaminker, père de Simone Signoret, et Andronikof n'étaient pas très chauds. Kaminker, soucieux de solennités, trouvait même qu'AMICI faisait un peu calembour. À ce moment, Andronikof me dit que «c'est encore trop tôt» et ce ne fut en effet qu'en 1953 que Kaminker, Jacob, Andronikof et moi-même convoquâmes la «Constituante».

\section{La IACIT}

Revenons à la «International Association of Conference Interpreters and Translators». Malgré nos efforts, nous n'avons guère trouvé de documents y afférents. Elle fut fondée à Londres peu après la guerre par Frank Barker, ancien de la Société des Nations qui ne voulut pas suivre ses collègues aux Nations Unies à New York. Teddy Pilley fut un des co-fondateurs. Mentionnons quelques-uns des membres interprètes les plus marquants de cette association: Roger Glémet, qui fit ses débuts dans la profession en 1946 à Londres, Nora Saxe et Louise O’Brien qui formèrent, avec Juanita Pickering, une des premières entreprises d'interprètes au nom de «OPS». On y trouve aussi Lia Calman, Hilde Watson, Helga Lund, Cleves Marett, ainsi que l'infatigable Laura Sutherland capable de travailler seule en simultanée pendant des heures. Nous ignorons qui parmi les autres interprètes de cette époque fit partie de la IACIT, tels que Philippe Picard, Ruth Hall, Elena Shapiro, le remuant Jean de Laforest, "monsieur motion d'ordre» comme nous l'appellerions plus tard à l'AIIC. N'en furent pas membres les deux éminents interprètes du gouvernement britannique, le colonel Birse et David Balfour qui avaient fait partie de la délégation du Royaume-Uni à Yalta et à Potsdam. La IACIT atteignit son apogée vers 1948-1950.

Le seul document en nos mains comportant des renseignements plus précis sur elle est le compte rendu d'une rencontre entre des représentants de la IACIT et de l'AIT qui se tint le 19 avril 1949 au célèbre Café de la Clémence à Genève à l'occasion de la Conférence diplomatique convoquée pour remettre à jour les Conventions de la CroixRouge. Côté IACIT, nous trouvons Frank Barker, président, Teddy Pilley, Letondot et Roger Glémet. L'AEEEI, comme l'AIT s'appelait encore, fut représentée par Véra Wegelin, présidente-fondatrice, G. John, vice-président, Mlles Vernet (de Morawitz), Hess, Runte, Hemmerich (Barter), Bouverat et MM. Berkovits et Keiser, rapporteur. Selon Frank Barker, la IACIT « est une association professionnelle équivalente à un syndicat sans toutefois être enregistrée comme tel. Elle fut fondée pour défendre les intérêts des interprètes et traducteurs membres, sans être un bureau de placement. Elle compte actuellement environ 250 membres répartis sur plusieurs pays.»

Les tarifs des interprètes et traducteurs de la IACIT sont:

3 guineas pour le «potential member».

4 guineas tarif minimum.

5 guineas tarif usuel pour les traducteurs. 
6-7 guineas tarif usuel pour les interprètes ou traducteurs-réviseurs.

Tous les tarifs sont «sans per diem».

(Une guinea correspondait à $\mathfrak{E} 1$.05. La livre sterling valait environ 20 francs suisses.)

On constate que les taux minima des deux associations sont comparables, mais que pour les interprètes de conférence l'AAEEI devrait légèrement augmenter le tarif. Teddy Pilley expose alors le but de la rencontre: une concertation entre les deux groupements en face de «l'offensive organisée contre les interprètes et qui, pour la première fois, s'est montrée lors d'une offre faite aux interprètes par l'OMS pour une conférence à Rome: 80 francs suisses, per diem compris!» Teddy Pilley souligne «la position actuelle forte des interprètes, vu la pénurie de personnes qualifiées. Sur les huit interprètes demandés, l'OMS n'en a trouvé que deux.» Roger Glémet propose que «tous les interprètes membres de la IACIT et de l'AAEEI refusent d'accepter les conditions offertes par l'OMS. On pourrait, du reste, calculer qu'en se basant sur le traitement annuel des interprètes permanents des organisations internationales, les honoraires pour les engagements temporaires ressortiraient à plus de 80 francs suisses sans per diem. » En conclusion de la rencontre, le principe d'une concertation fut approuvé et peu après, le taux d'honoraires passa à US\$ 25 (env. 110 francs suisses).

Pour la petite histoire: ce taux fut très coriace et la lutte pour faire sauter le double verrou honoraires-per diem fut longue puisque, selon Roger Glémet, «en 1958 on en était toujours à US\$ 25 (taux free-lance des Nations unies) et au per diem, fixé par Lethbridge, de US\$ 4. Grâce à l'appui interne, mais ferme, d'Armstrong, chef de l'Administration de l'OMS - et de Barnes, son homologue à l'UNESCO (note de l'auteur) - les deux boulons sautèrent et tout, par la suite, fut plus facile, d'autant que l'enfant AIIC croissait en force et en sagesse. »

Pour revenir à la IACIT, dès 1951 on n'en entend plus guère parler. Sa fin rapide peut être attribuée à trois facteurs:

- La difficulté d'assumer simultanément la défense des intérêts des interprètes et des traducteurs de conférence. Les derniers mirent du temps à s'organiser. En effet, ce ne fut qu'en 1962 que naquit l'Association internationale des traducteurs de conférence (AITC).

- La trop forte centralisation de la IACIT sur Londres qui, après le départ des Nations unies à New York et l'installation de plusieurs institutions spécialisées à Genève, Paris et Rome, avait perdu du poids en tant que centre de conférences internationales.

- Les discussions en vue de la création de l'AIIC dont le siège allait se trouver à Paris.

\section{La LACI}

Pour combler le vide, nos collègues londoniens décidèrent de fonder une association centrée essentiellement sur Londres et le Royaume-Uni, la London Association of Conference Interpreters (LACI). Une lettre de Geoffrey D.M. Block, annonçant à l'AIT le lancement de cette Association, en décrit bien la nature et met en évidence un problème qui allait devenir une sorte de serpent de mer au sein de l'AIIC, celui du respect des conditions locales par des interprètes venant d'autres pays. Voici quelques extraits de cette lettre:

At a meeting of the Association held on July 24th,1951, it was decided that I should write to inform you of the formation of the London Association of Conference Interpreters. This Association whose main object is to protect and enhance the status of 
Conference Interpreters includes among its members the great majority of the UK interpreters practising at international conferences. It is felt that our colleagues abroad will be interested to learn of the formation of this Association.

I have also been asked to let you know that this Association resolved that (subject to a few exceptions to cover existing commitments of long standing) the minimum to be accepted by any member of the Association should be $£ 8.80$ per day. It is thought that our colleagues abroad may care to have this minimum in mind when considering conferences in this country.

I am sure that you will appreciate that it will be to the advantage of interpreters both here and abroad if the same minimum fee is used for this country by all of us and lower fees are not accepted by interpreters from abroad for work in the United Kingdom...

La LACI fut très active pendant de nombreuses années, poussa à la double-appartenance LACI-AIIC et joua un rôle capital lorsqu'il s'agit de faire réussir le «Gentlemen's Agreement» Paris-Genève-Londres dont il sera question plus loin. Comme l'AIT à Genève, elle s'aligna sur l'AIIC en matière de tarifs et de conditions de travail tout en tenant compte de certaines particularités du marché britannique. Nous ignorons les raisons et la date de sa cessation d'activité.

\section{La FLIG}

La FLIG (Free-Lance Interpreters Geneva) fut fondée le 23 janvier 1951 par un groupe d'interprètes travaillant essentiellement pour les institutions intergouvernementales. Elle se consolida en adoptant ses statuts lors de l'Assemblée générale du 21 mars 1951. Son bureau se composait de P. L. Artzrouni, Norbert Berger et Pierre Lambert. Elle se définit comme "Groupement de coordination entre interprètes et de coopération avec les organisations internationales». En fait, il s'agissait surtout de réagir à l'aggravation de la situation des interprètes free-lance travaillant auprès de l'Office européen des Nations unies et des institutions spécialisées sises à Genève: honoraires bloqués à $\$ 25$, per diem variant entre $\$ 4$ et, exceptionnellement, $\$ 7$, comparé au per diem de $\$ 12.50$ des interprètes fonctionnaires en mission.

Les négociations entre la FLIG et le porte-parole des institutions des Nations unies étaient souvent très serrées, le point fort des interprètes étant le «seller's market» favorable, le point faible le manque de coordination et d'esprit de lutte en face de la dureté de M. Lethbridge, secrétaire du Comité consultatif pour les questions administratives (CCQA) et principal négociateur du côté des institutions. Un rapport de Pierre Lambert de mars 1952 montre bien cela, tout en mettant en évidence un certain nombre d'ébauches de solutions que nous retrouverons dix ans plus tard lors de la négociation des premiers accords avec les trois familles d'organisations, dont l'assurance maladie, le paiement complet des jours de voyage, l'assimilation des free-lance aux permanents en matière de per diem et d'indexation de la rémunération. D'autres «benefits", suggérés par les organisations, n’ont jamais été retenus, comme par exemple les journées garanties payées pour l'étude de documents et des congés payés pendant les longues conférences.

Quant à la position de la FLIG en face de la perspective de la création de l'AIIC, certains dirigeants et quelques interprètes «monoculturistes» auraient sans doute préféré une extension d'un groupement de type FLIG à toute la famille des Nations unies. D'autres, notamment Erich Longet, qui avait remplacé Artzrouni au bureau, 
estimaient que seule une organisation professionnelle représentative de tous les interprètes et de tous les circuits avait des chances de faire le poids vis-à-vis des employeurs. Finalement, la FLIG se rallia à la position adoptée avant elle par l'AIT, soit de recommander l'adhésion au «Gentlemen's Agreement». Après la fondation de l'AIIC, la FLIG se mit en veilleuse pour disparaître en 1955.

\section{Le Gentlemen's Agreement}

À l'issue de l'Assemblée constitutive du 11 novembre 1953, l'AIIC comptait 35 membres, dont une vingtaine domiciliés en France. Si l'Association voulait éviter le piège de géocentrisme rencontré par la IACIT, mais surtout si elle voulait se faire entendre par les grands employeurs, il était absolument indispensable d'étendre son rayonnement géographique et notamment de renforcer sa présence à Genève et à Londres. Or, à Genève surtout, l'organisation de la profession avait fait de sérieux progrès et l'enthousiasme pour le «machin parisien» n'était pas évident. À Londres aussi, certains avaient le sentiment qu'on avait enterré un peu trop vite la IACIT en faveur de la future AIIC. Surtout, on n'aimait pas l'idée de devoir se soumettre à une procédure d'admission sélective.

À l'Assemblée de Paris de 1955, le débat sur les moyens de faire l'unité de la profession, géographiquement et entre interprètes permanents et free-lance, fut sincère mais acharné. Londres voyait d'abord une fédération d'associations locales, à Genève on cherchait l'unité de la profession, mais on voulait s'assurer que les décisions concernant les free-lance réuniraient au moins l'appui de la majorité de membres freelance - il était même question de faire prendre ces décisions par une Commission des free-lance de l'AIIC. Pour l'admission des candidats, il fut proposé de supprimer le «barrage Conseil» dans la procédure d'admission. Finalement, l'Assemblée créa une Commission tripartite Londres (Cleves Marett et Nora Saxe), Genève (Elisabeth Meister et Wadi Keiser) et Paris (Constantin Andronifkof et Marie-France Skuncke) pour discuter les modalités de cet arrangement, mais surtout pour travailler le terrain et préparer un climat favorable. D'où l'idée, lancée après l'Assemblée de 1955 par Wadi Keiser, élu entre-temps président de l'AIT, de se mettre d'accord sur un «Gentlemen's Agreement» en vertu duquel tous les interprètes de conférence qui présenteraient leur candidature à l'Assemblée de Genève de 1956 seraient admis sans examen quant au fond (langues, "péchés du passé», etc.). L'idée fut acceptée par la Commission tripartite et par le Conseil et largement débattue dans les trois centres. À Genève, où l'hésitation était la plus forte, une réunion fut convoquée sur l'initiative de l'AIT pour le 12 janvier 1956. Le rapport de cette réunion mérite d'être reproduit:

Association d'Interprètes

Genève, février 1956

et de Traducteurs (AIT)

Décision prises par les interprètes réunis à Genève le 12 janvier 1956

Une trentaine d'interprètes de Genève, Lausanne et Zürich, membres et non-membres de l'Association Internationale des Interprètes de Conférence, réunis le 12 janvier 1956, ont décidé, à l'unanimité des voix contre trois abstentions, de recommander à tous les interprètes de conférence professionnels ayant leur domicile habituel en Suisse d'adhérer à l'AIIC. 
Cette décision est intervenue compte tenu du nouveau projet de Statuts de l'AIIC, élaboré par la réunion tripartite (Paris/Londres/Genève) qui s'est tenue à Paris le 18 décembre 1955.

Elle est intervenue aussi sur la base du "Gentlemen's Agreement» conclu entre l'AIIC et les interprètes de Genève et de Londres visant à ce que tous les interprètes professionnels des deux centres qui feraient en ce moment-ci leur demande d'adhésion seraient effectivement admis comme nouveaux membres, à la condition, bien entendu, d'être des interprètes professionnels qualifiés et de remplir les conditions de forme présidant à l'admission de nouveaux membres et prévues dans les Statuts de l'AIIC (présentation par 5 membres, dont un du Conseil).

Nous joignons à cette circulaire un exemplaire du nouveau projet de Statuts (qui devra être ratifié par la prochaine Assemblée) et une formule de demande d'admission.

Ceux parmi nos collègues qui décideront d'adhérer à l'AIIC, conformément à la recommandation ci-dessus, sont priés, ou bien d'envoyer la formule de demande d'admission directement au président de l'AIIC (Monsieur Hans Jacob, 32 avenue du Roule, Neuilly/Seine) et d'en aviser le soussigné, ou bien (notamment s'ils ne connaissent pas un nombre suffisant de "parrains») d'envoyer la demande dûment remplie à M. Walter Keiser (67, bd de la Cluse, Genève) qui se fera fort de leur trouver le nombre nécessaire de "parrains» et qui transmettra ensuite les demandes au président de l'AIIC.

Il importe, en vue de l'observation du "Gentlemen's Agreement», que nous soyons exactement mis au courant de toutes les demandes d'admission, que celles-ci se fassent directement ou par notre intermédiaire.

L'admission de ces nouveaux membres sera un des premiers points à l'ordre du jour de la prochaine Assemblée. Ils pourront donc y participer de plein droit.

D'après les dernières informations dont nous disposons de nos collègues de Londres, 13 sur 17 membres de LACI ont proposé leur candidature.

La cotisation annuelle est de $\$ 15$.

Nous prions tous nos collègues de nous faire part de leur décision dans un délai d'une semaine à partir de la réception de cette circulaire.

P.S. Étant donné que nous ne disposons pas des adresses de tous les interprètes de conférence de Suisse, nous prions nos collègues de se mettre en rapport avec tous les autres collègues qu'ils connaissent et de leur demander de nous envoyer leur adresse. Ils recevront alors les mêmes documents.

L'Assemblée 1956 de l'AIIC se tiendra à Genève les 24/25 mars 1956. Raison de plus pour que nous y soyons nombreux.

Le «Gentlemen's Agreement » porta ses fruits. Le résultat escompté fut atteint. Les chiffres sont éloquents:

- Assemblée 1953: 35 membres, dont 2 de Londres, 2 de Genève

- Assemblée 1955: 83 membres, dont 2 de Londres, 21 de Suisse

- Assemblée 1956: 153 membres, dont 15 du Royaume-Uni, 41 de Suisse.

Nous avons ainsi vu le monde de l'interprétation de conférence de l'après-guerre immédiat, rencontré des personnalités marquant cette époque, assisté au développement de marchés et de circuits avec leurs structures et conditions de travail, observé la naissance des premières organisations professionnelles et leur concours à la création de l'AIIC. Nous espérons avoir ainsi éveillé l'intérêt pour la suite de cette rétrospective historique, qui sera publiée ultérieurement sous la houlette de l'AIIC. 